\title{
Holographic fermions in external magnetic fields
}

\author{
E. Gubankova, , , J. Brill, ${ }^{2}$ M. Cubrović, ${ }^{2}$ K. Schalm, ${ }^{2}$ P. Schijven, ${ }^{2, \text { 甲 }}$ and J. Zaanen ${ }^{2}$ \\ ${ }^{1}$ Institute for Theoretical Physics, J. W. Goethe-University, \\ D-60438 Frankfurt am Main, Germany \\ ${ }^{2}$ Instituut Lorentz, Leiden University, Niels Bohrweg 2, \\ 2300 RA Leiden, Netherlands
}

\begin{abstract}
We study the Fermi level structure of $2+1$-dimensional strongly interacting electron systems in external magnetic field using the AdS/CFT correspondence. The gravity dual of a finite density fermion system is a Dirac field in the background of the dyonic AdS-Reissner-Nordström black hole. In the probe limit the magnetic system can be reduced to the non-magnetic one, with Landau-quantized momenta and rescaled thermodynamical variables. We find that at strong enough magnetic fields, the Fermi surface vanishes and the quasiparticle is lost either through a crossover to conformal regime or through a phase transition to an unstable Fermi surface. In the latter case, the vanishing Fermi velocity at the critical magnetic field triggers the non-Fermi liquid regime with unstable quasiparticles and a change in transport properties of the system. We associate it with a metal-"strange metal" phase transition. Next we compute compute the DC Hall and longitudinal conductivities using the gravity-dressed fermion propagators. For dual fermions with a large charge, many different Fermi surfaces contribute and the Hall conductivity is quantized as expected for integer Quantum Hall Effect (QHE). At strong magnetic fields, as additional Fermi surfaces open up, new plateaus typical for the fractional QHE appear. The somewhat irregular pattern in the length of fractional QHE plateaus resemble the outcomes of experiments on thin graphite in a strong magnetic field. Finally, motivated by the absence of the sign problem in holography, we suggest a lattice approach to the AdS calculations of finite density systems.
\end{abstract}

Keywords: AdS/CFT, strongly correlated electrons, transport, lattice

\section{INTRODUCTION}

The study of strongly interacting fermionic systems at finite density and temperature is a challenging task in condensed matter and high energy physics. Analytical methods are limited or not available for strongly coupled systems, and numerical simulation of fermions at finite density breaks down because of the sign problem [1]. There has been an increased activity in describing finite density fermionic matter by a gravity dual using the holographic AdS/CFT correspondence [2]. The gravitational solution dual to the finite chemical potential system is the electrically charged AdS-Reissner-Nordström (RN) black hole, which provides a background where only the metric and Maxwell fields are nontrivial and all matter fields vanish. In the classical gravity limit, the decoupling of the Einstein-Maxwell sector holds and leads to universal results, which is an appealing feature of applied holography. Indeed, the celebrated result for the ratio of the shear viscosity over the entropy density [3] is identical for many strongly interacting theories and has been considered a robust prediction of the AdS/CFT correspondence.

However, an extremal black hole alone is not enough to describe finite density systems as it does not source the matter fields. In holography, at leading order, the Fermi surfaces are not evident in the gravitational geometry, but can only be detected by external probes; either probe D-branes [2] or probe bulk fermions [4 7]. Here we shall consider the latter option, where the free Dirac field in the bulk carries a finite charge density [8]. We ignore electromagnetic and gravitational backreaction of the charged fermions on the bulk spacetime geometry (probe approximation). At large temperatures, $T \gg \mu$, this approach provides a reliable hydrodynamic description of transport at a quantum criticality (in the vicinity of superfluid-insulator transition) [9]. At small temperatures, $T \ll \mu$, in some cases sharp Fermi surfaces emerge with either conventional Fermi-liquid scaling [5] or of a non-Fermi liquid type [6] with scaling properties that differ significantly from those predicted by the Landau Fermi liquid theory. The non-trivial scaling behavior of these non-Fermi liquids has been studied semi-analytically in 7] and is of great interest as high- $T_{c}$ superconductors and metals near the critical point are believed to represent non-Fermi liquids.

\footnotetext{
*Also at ITEP, Moscow, Russia.

${ }^{\dagger}$ Address from October 1st: Albert-Ludwigs-Universität Freiburg D-79104 Freiburg, Germany.
} 
What we shall study is the effects of magnetic field on the holographic fermions. A magnetic field is a probe of finite density matter at low temperatures, where the Landau level physics reveals the Fermi level structure. The gravity dual system is described by a AdS dyonic black hole with electric and magnetic charges $Q$ and $H$, respectively, corresponding to a $2+1$-dimensional field theory at finite chemical potential in an external magnetic field [10]. Probe fermions in the background of the dyonic black hole have been considered in [11, 12]; and probe bosons in the same background have been studied in [13]. Quantum magnetism is considered in [14].

The Landau quantization of momenta due to the magnetic field found there, shows again that the AdS/CFT correspondence has a powerful capacity to unveil that certain quantum properties known from quantum gases have a much more ubiquitous status than could be anticipated theoretically. A first highlight is the demonstration [15] that the Fermi surface of the Fermi gas extends way beyond the realms of its perturbative extension in the form of the Fermi-liquid. In AdS/CFT it appears to be gravitationally encoded in the matching along the scaling direction between the 'bare' Dirac waves falling in from the 'UV' boundary, and the true IR excitations living near the black hole horizon. This IR physics can insist on the disappearance of the quasiparticle but, if so, this 'critical Fermi-liquid' is still organized 'around' a Fermi surface. The Landau quantization, the organization of quantum gaseous matter in quantized energy bands (Landau levels) in a system of two space dimensions pierced by a magnetic field oriented in the orthogonal spatial direction, is a second such quantum gas property. We shall describe here following [11], that despite the strong interactions in the system, the holographic computation reveals the same strict Landau-level quantization. Arguably, it is the mean-field nature imposed by large $N$ limit inherent in AdS/CFT that explains this. The system is effectively non-interacting to first order in $1 / N$. The Landau quantization is not manifest from the geometry, but as we show this statement is straightforwardly encoded in the symmetry correspondences associated with the conformal compactification of $A d S$ on its flat boundary (i. e., in the UV CFT).

An interesting novel feature in strongly coupled systems arises from the fact that the background geometry is only sensitive to the total energy density $Q^{2}+H^{2}$ contained in the electric and magnetic fields sourced by the dyonic black hole. Dialing up the magnetic field is effectively similar to a process where the dyonic black hole loses its electric charge. At the same time, the fermionic probe with charge $q$ is essentially only sensitive to the Coulomb interaction $g q Q$. As shown in [11], one can therefore map a magnetic to a non-magnetic system with rescaled parameters (chemical potential, fermion charge) and same symmetries and equations of motion, as long as the Reissner-Nordström geometry is kept.

Translated to more experiment-compatible language, the above magnetic-electric mapping means that the spectral functions at nonzero magnetic field $h$ are identical to the spectral function at $h=0$ for a reduced value of the coupling constant (fermion charge) $q$, provided the probe fermion is in a Landau level eigenstate. A striking consequence is that the spectrum shows conformal invariance for arbitrarily high magnetic fields, as long as the system is at negligible to zero density. Specifically, a detailed analysis of the fermion spectral functions reveals that at strong magnetic fields the Fermi level structure changes qualitatively. There exists a critical magnetic field at which the Fermi velocity vanishes. Ignoring the Landau level quantization, we show that this corresponds to an effective tuning of the system from a regular Fermi liquid phase with linear dispersion and stable quasiparticles to a non-Fermi liquid liquid with fractional power law dispersion and unstable excitations. This phenomenon can be interpreted as a transition from metallic phase to a "strange metal" at the critical magnetic field and corresponds to the change of the infrared conformal dimension from $\nu>1 / 2$ to $\nu<1 / 2$ while the Fermi momentum stays nonzero and the Fermi surface survives. Increasing the magnetic field further, this transition is followed by a "strange-metal"-conformal crossover and eventually, for very strong fields, the system always has near-conformal behavior where $k_{F}=0$ and the Fermi surface disappears.

For some Fermi surfaces, this surprising metal-"strange metal" transition is not physically relevant as the system prefers to directly enter the conformal phase. Whether a fine tuned system exists that does show a quantum critical phase transition from a FL to a non-FL is determined by a Diophantine equation for the Landau quantized Fermi momentum as a function of the magnetic field. Perhaps these are connected to the magnetically driven phase transition found in $\mathrm{AdS}_{5} / \mathrm{CFT}_{4}[16$. We leave this subject for further work.

Overall, the findings of Landau quantization and "discharge" of the Fermi surface are in line with the expectations: both phenomena have been found in a vast array of systems [17] and are almost tautologically tied to the notion of a Fermi surface in a magnetic field. Thus we regard them also as a sanity check of the whole bottom-up approach of fermionic AdS/CFT [4 6, 15], giving further credit to the holographic Fermi surfaces as having to do with the real world.

Next we use the information of magnetic effects the Fermi surfaces extracted from holography to calculate the quantum Hall and longitudinal conductivities. Generally speaking, it is difficult to calculate conductivity holographically beyond the Einstein-Maxwell sector, and extract the contribution of holographic fermions. In the semiclassical approximation, one-loop corrections in the bulk setup involving charged fermions have been calculated [15]. In another approach, the backreaction of charged fermions on the gravity-Maxwell sector has been taken into account and incorporated in calculations of the electric conductivity [8]. We calculate the one-loop contribution on the CFT side, 
which is equivalent to the holographic one-loop calculations as long as vertex corrections do not modify physical dependencies of interest [15, 18]. As we dial the magnetic field, the Hall plateau transition happens when the Fermi surface moves through a Landau level. One can think of a difference between the Fermi energy and the energy of the Landau level as a gap, which vanishes at the transition point and the 2+1-dimensional theory becomes scale invariant. In the holographic D3-D7 brane model of the quantum Hall effect, plateau transition occurs as D-branes move through one another [19]. In the same model, a dissipation process has been observed as D-branes fall through the horizon of the black hole geometry, that is associated with the quantum Hall insulator transition. In the holographic fermion liquid setting, dissipation is present through interaction of fermions with the horizon of the black hole. We have also used the analysis of the conductivities to learn more about the metal-strange metal phase transition as well as the crossover back to the conformal regime at high magnetic fields.

We conclude with the remark that the findings summarized above are in fact somewhat puzzling when contrasted to the conventional picture of quantum Hall physics. It is usually stated that the quantum Hall effect requires three key ingredients: Landau quantization, quenched disorder ${ }^{1}$ and (spatial) boundaries, i. e., a finite-sized sample [20]. The first brings about the quantization of conductivity, the second prevents the states from spilling between the Landau levels ensuring the existence of a gap and the last one in fact allows the charge transport to happen (as it is the boundary states that actually conduct). In our model, only the first condition is satisfied. The second is put by hand by assuming that the gap is automatically preserved, i. e. that there is no mixing between the Landau levels. There is, however, no physical explanation as to how the boundary states are implicitly taken into account by AdS/CFT.

The paper is organized as follows. We outline the holographic setting of the dyonic black hole geometry and bulk fermions in the section II In section [II we prove the conservation of conformal symmetry in the presence of the magnetic fields. Section IV] is devoted to the holographic fermion liquid, where we obtain the Landau level quantization, followed by a detailed study of the Fermi surface properties at zero temperature in section $\mathrm{V}$. We calculate the DC conductivities in section VI and compare the results with available data in graphene. In section VII. we show that the fermion sign problem is absent in the holographic setting, therefore allowing lattice simulations of finite density matter in principle.

\section{HOLOGRAPHIC FERMIONS IN A DYONIC BLACK HOLE}

We first describe the holographic setup with the dyonic black hole, and the dynamics of Dirac fermions in this background. In this paper, we exclusively work in the probe limit, i. e., in the limit of large fermion charge $q$.

\section{A. Dyonic black hole}

We consider the gravity dual of 3-dimensional conformal field theory (CFT) with global $U(1)$ symmetry. At finite charge density and in the presence of magnetic field, the system can be described by a dyonic black hole in 4dimensional anti-de Sitter space-time, $A d S_{4}$, with the current $J_{\mu}$ in the CFT mapped to a $U(1)$ gauge field $A_{M}$ in $A d S$. We use $\mu, \nu, \rho, \ldots$ for the spacetime indices in the CFT and $M, N, \ldots$ for the global spacetime indices in $A d S$.

The action for a vector field $A_{M}$ coupled to $A d S_{4}$ gravity can be written as

$$
S_{g}=\frac{1}{2 \kappa^{2}} \int d^{4} x \sqrt{-g}\left(\mathcal{R}+\frac{6}{R^{2}}-\frac{R^{2}}{g_{F}^{2}} F_{M N} F^{M N}\right),
$$

where $g_{F}^{2}$ is an effective dimensionless gauge coupling and $R$ is the curvature radius of $A d S_{4}$. The equations of motion following from eq. (11) are solved by the geometry corresponding to a dyonic black hole, having both electric and magnetic charge:

$$
d s^{2}=g_{M N} d x^{M} d x^{N}=\frac{r^{2}}{R^{2}}\left(-f d t^{2}+d x^{2}+d y^{2}\right)+\frac{R^{2}}{r^{2}} \frac{d r^{2}}{f} .
$$

The redshift factor $f$ and the vector field $A_{M}$ reflect the fact that the system is at a finite charge density and in an

\footnotetext{
1 Quenched disorder means that the dynamics of the impurities is "frozen", i. e. they can be regarded as having infinite mass. When coupled to the Fermi liquid, they ensure that below some scale the system behaves as if consisting of non-interacting quasiparticles only.
} 
external magnetic field:

$$
\begin{aligned}
f & =1+\frac{Q^{2}+H^{2}}{r^{4}}-\frac{M}{r^{3}} \\
A_{t} & =\mu\left(1-\frac{r_{0}}{r}\right), \quad A_{y}=h x, \quad A_{x}=A_{r}=0
\end{aligned}
$$

where $Q$ and $H$ are the electric and magnetic charge of the black hole, respectively. Here we chose the Landau gauge; the black hole chemical potential $\mu$ and the magnetic field $h$ are given by

$$
\mu=\frac{g_{F} Q}{R^{2} r_{0}}, \quad h=\frac{g_{F} H}{R^{4}},
$$

with $r_{0}$ is the horizon radius determined by the largest positive root of the redshift factor $f\left(r_{0}\right)=0$ :

$$
M=r_{0}^{3}+\frac{Q^{2}+H^{2}}{r_{0}} .
$$

The boundary of the $A d S$ is reached for $r \rightarrow \infty$. The geometry described by eqs. (2) describes the boundary theory at finite density, i. e., a system in a charged medium at the chemical potential $\mu=\mu_{\mathrm{bh}}$ and in transverse magnetic field $h=h_{\mathrm{bh}}$, with charge, energy, and entropy densities given, respectively, by

$$
\rho=2 \frac{Q}{\kappa^{2} R^{2} g_{F}}, \quad \epsilon=\frac{M}{\kappa^{2} R^{4}}, \quad s=\frac{2 \pi}{\kappa^{2}} \frac{r_{0}^{2}}{R^{2}} .
$$

The temperature of the system is identified with the Hawking temperature of the black hole, $T_{H} \sim\left|f^{\prime}\left(r_{0}\right)\right| / 4 \pi$,

$$
T=\frac{3 r_{0}}{4 \pi R^{2}}\left(1-\frac{Q^{2}+H^{2}}{3 r_{0}^{4}}\right) .
$$

Since $Q$ and $H$ have dimensions of $[L]^{2}$, it is convenient to parametrize them as

$$
Q^{2}=3 r_{*}^{4}, Q^{2}+H^{2}=3 r_{* *}^{4}
$$

In terms of $r_{0}, r_{*}$ and $r_{* *}$ the above expressions become

$$
f=1+\frac{3 r_{* *}^{4}}{r^{4}}-\frac{r_{0}^{3}+3 r_{* *}^{4} / r_{0}}{r^{3}},
$$

with

$$
\mu=\sqrt{3} g_{F} \frac{r_{*}^{2}}{R^{2} r_{0}}, \quad h=\sqrt{3} g_{F} \frac{\sqrt{r_{* *}^{4}-r_{*}^{4}}}{R^{4}} .
$$

The expressions for the charge, energy and entropy densities, as well as for the temperature are simplified as

$$
\begin{aligned}
\rho & =\frac{2 \sqrt{3}}{\kappa^{2} g_{F}} \frac{r_{*}^{2}}{R^{2}}, \epsilon=\frac{1}{\kappa^{2}} \frac{r_{0}^{3}+3 r_{* *}^{4} / r_{0}}{R^{4}}, s=\frac{2 \pi}{\kappa^{2}} \frac{r_{0}^{2}}{R^{2}}, \\
T & =\frac{3}{4 \pi} \frac{r_{0}}{R^{2}}\left(1-\frac{r_{* *}^{4}}{r_{0}^{4}}\right) .
\end{aligned}
$$

In the zero temperature limit, i. e., for an extremal black hole, we have

$$
T=0 \rightarrow r_{0}=r_{* *},
$$

which in the original variables reads $Q^{2}+H^{2}=3 r_{0}^{4}$. In the zero temperature limit (12), the redshift factor $f$ as given by eq. (9) develops a double zero at the horizon:

$$
f=6 \frac{\left(r-r_{* *}\right)^{2}}{r_{* *}^{2}}+\mathcal{O}\left(\left(r-r_{* *}\right)^{3}\right)
$$


As a result, near the horizon the $A d S_{4}$ metric reduces to $A d S_{2} \times \mathbb{R}^{2}$ with the curvature radius of $A d S_{2}$ given by

$$
R_{2}=\frac{1}{\sqrt{6}} R
$$

This is a very important property of the metric, which considerably simplifies the calculations, in particular in the magnetic field.

In order to scale away the $A d S_{4}$ radius $R$ and the horizon radius $r_{0}$, we introduce dimensionless variables

$$
\begin{aligned}
& r \rightarrow r_{0} r, \quad r_{*} \rightarrow r_{0} r_{*}, \quad r_{* *} \rightarrow r_{0} r_{* *}, \\
& M \rightarrow r_{0}^{3} M, Q \rightarrow r_{0}^{2} Q, \quad, H \rightarrow r_{0}^{2} H
\end{aligned}
$$

and

$$
\begin{aligned}
& (t, \vec{x}) \rightarrow \frac{R^{2}}{r_{0}}(t, \vec{x}), \quad A_{M} \rightarrow \frac{r_{0}}{R^{2}} A_{M}, \omega \rightarrow \frac{r_{0}}{R^{2}} \omega, \\
& \mu \rightarrow \frac{r_{0}}{R^{2}} \mu, \quad h \rightarrow \frac{r_{0}^{2}}{R^{4}} h, \quad T \rightarrow \frac{r_{0}}{R^{2}} T, \\
& d s^{2} \rightarrow R^{2} d s^{2} .
\end{aligned}
$$

Note that the scaling factors in the above equation that describes the quantities of the boundary field theory involve the curvature radius of $A d S_{4}$, not $A d S_{2}$.

In the new variables we have

$$
\begin{aligned}
& T=\frac{3}{4 \pi}\left(1-r_{* *}^{4}\right)=\frac{3}{4 \pi}\left(1-\frac{Q^{2}+H^{2}}{3}\right), \quad f=1+\frac{3 r_{* *}^{4}}{r^{4}}-\frac{1+3 r_{* *}^{4}}{r^{3}} \\
& A_{t}=\mu\left(1-\frac{1}{r}\right), \quad \mu=\sqrt{3} g_{F} r_{*}^{2}=g_{F} Q, \quad h=g_{F} H
\end{aligned}
$$

and the metric is given by

$$
d s^{2}=r^{2}\left(-f d t^{2}+d x^{2}+d y^{2}\right)+\frac{1}{r^{2}} \frac{d r^{2}}{f},
$$

with the horizon at $r=1$, and the conformal boundary at $r \rightarrow \infty$.

At $T=0, r_{* *}$ becomes unity, and the redshift factor develops the double zero near the horizon,

$$
f=\frac{(r-1)^{2}\left(r^{2}+2 r+3\right)}{r^{4}} .
$$

As mentioned before, due to this fact the metric near the horizon reduces to $A d S_{2} \times \mathbb{R}^{2}$ where the analytical calculations are possible for small frequencies [7]. However, in the chiral limit $m=0$, analytical calculations are also possible in the bulk $A d S_{4}$ [21], which we utilize in this paper.

\section{B. Holographic fermions}

To include the bulk fermions, we consider a spinor field $\psi$ in the $A d S_{4}$ of charge $q$ and mass $m$, which is dual to an operator $\mathcal{O}$ in the boundary $C F T_{3}$ of charge $q$ and dimension

$$
\Delta=\frac{3}{2}+m R
$$

with $m R \geq-\frac{1}{2}$ and in dimensionless units corresponds to $\Delta=\frac{3}{2}+m$. In the black hole geometry, eq. (2), the quadratic action for $\psi$ reads as

$$
S_{\psi}=i \int d^{4} x \sqrt{-g}\left(\bar{\psi} \Gamma^{M} \mathcal{D}_{M} \psi-m \bar{\psi} \psi\right)
$$

where $\bar{\psi}=\psi^{\dagger} \Gamma^{t}$, and

$$
\mathcal{D}_{M}=\partial_{M}+\frac{1}{4} \omega_{a b M} \Gamma^{a b}-i q A_{M}
$$


where $\omega_{a b M}$ is the spin connection, and $\Gamma^{a b}=\frac{1}{2}\left[\Gamma^{a}, \Gamma^{b}\right]$. Here, $M$ and $a, b$ denote the bulk space-time and tangent space indices respectively, while $\mu, \nu$ are indices along the boundary directions, i. e. $M=(r, \mu)$. Gamma matrix basis (Minkowski signature) is given by eq. (A12) as in 7].

We will be interested in spectra and response functions of the boundary fermions in the presence of magnetic field. This requires solving the Dirac equation in the bulk [5, 6]:

$$
\left(\Gamma^{M} \mathcal{D}_{M}-m\right) \psi=0 .
$$

From the solution of the Dirac equation at small $\omega$, an analytic expression for the retarded fermion Green's function of the boundary CFT at zero magnetic field has been obtained in [7]. Near the Fermi surface it reads as [7]:

$$
G_{R}(\Omega, k)=\frac{\left(-h_{1} v_{F}\right)}{\omega-v_{F} k_{\perp}-\Sigma(\omega, T)},
$$

where $k_{\perp}=k-k_{F}$ is the perpendicular distance from the Fermi surface in momentum space, $h_{1}$ and $v_{F}$ are real constants calculated below, and the self-energy $\Sigma=\Sigma_{1}+i \Sigma_{2}$ is given by [7]

$$
\Sigma(\omega, T) / v_{F}=T^{2 \nu} g\left(\frac{\omega}{T}\right)=(2 \pi T)^{2 \nu} h_{2} \mathrm{e}^{i \theta-i \pi \nu} \frac{\Gamma\left(\frac{1}{2}+\nu-\frac{i \omega}{2 \pi T}+\frac{i \mu_{q}}{6}\right)}{\Gamma\left(\frac{1}{2}-\nu-\frac{i \omega}{2 \pi T}+\frac{i \mu_{q}}{6}\right)},
$$

where $\nu$ is the zero temperature conformal dimension at the Fermi momentum, $\nu \equiv \nu_{k_{F}}$, given by eq. (58), $\mu_{q} \equiv \mu q$, $h_{2}$ is a positive constant and the phase $\theta$ is such that the poles of the Green's function are located in the lower half of the complex frequency plane. These poles correspond to quasinormal modes of the Dirac Equation (23) and they can be found numerically solving $F\left(\omega_{*}\right)=0$ [22], with

$$
F(\omega)=\frac{k_{\perp}}{\Gamma\left(\frac{1}{2}+\nu-\frac{i \omega}{2 \pi T}+\frac{i \mu_{q}}{6}\right)}-\frac{h_{2} \mathrm{e}^{i \theta-i \pi \nu}(2 \pi T)^{2 \nu}}{\Gamma\left(\frac{1}{2}-\nu-\frac{i \omega}{2 \pi T}+\frac{i \mu_{q}}{6}\right)},
$$

The solution gives the full motion of the quasinormal poles $\omega_{*}^{(n)}\left(k_{\perp}\right)$ in the complex $\omega$ plane as a function of $k_{\perp}$. It has been found in [7, 22], that, if the charge of the fermion is large enough compared to its mass, the pole closest to the real $\omega$ axis bounces off the axis at $k_{\perp}=0$ (and $\omega=0$ ). Such behavior is identified with the existence of the Fermi momentum $k_{F}$ indicative of an underlying strongly coupled Fermi surface.

At $T=0$, the self-energy becomes $T^{2 \nu} g(\omega / T) \rightarrow c_{k} \omega^{2 \nu}$, and the Green's function obtained from the solution to the Dirac equation reads [7]

$$
G_{R}(\Omega, k)=\frac{\left(-h_{1} v_{F}\right)}{\omega-v_{F} k_{\perp}-h_{2} v_{F} \mathrm{e}^{i \theta-i \pi \nu} \omega^{2 \nu}},
$$

where $k_{\perp}=\sqrt{k^{2}}-k_{F}$. The last term is determined by the IR $A d S_{2}$ physics near the horizon. Other terms are determined by the UV physics of the $A d S_{4}$ bulk.

The solutions to (23) have been studied in detail in [5 77. Here we simply summarize the novel aspects due to the background magnetic field (formal details can be found in the Appendix).

- The background magnetic field $h$ introduces a discretization of the momentum (see Appendix A for details):

$$
k \rightarrow k_{\text {eff }}=\sqrt{2|q h| l}, \text { with } l \in N,
$$

with Landau level index $l[12$, 22]. These discrete values of $k$ are the analogue of the well-known Landau levels that occur in magnetic systems.

- There exists a (non-invertible) mapping on the level of Green's functions, from the magnetic system to the non-magnetic one by sending

$$
(H, Q, q) \mapsto\left(0, \sqrt{Q^{2}+H^{2}}, q \sqrt{1-\frac{H^{2}}{Q^{2}+H^{2}}}\right) .
$$

The Green's functions in a magnetic system are thus equivalent to those in the absence of magnetic fields. To better appreciate that, we reformulate eq. (29) in terms of the boundary quantities:

$$
\left(h, \mu_{q}, T\right) \mapsto\left(0, \mu_{q}, T\left(1-\frac{h^{2}}{12 \mu^{2}}\right)\right),
$$


where we used dimensionless variables defined in eqs. (1517). The magnetic field thus effectively decreases the coupling constant $q$ and increases the chemical potential $\mu=g_{F} Q$ such that the combination $\mu_{q} \equiv \mu q$ is preserved [11]. This is an important point as the equations of motion actually only depend on this combination and not on $\mu$ and $q$ separately [11]. In other words, eq. (30) implies that the additional scale brought about by the magnetic field can be understood as changing $\mu$ and $T$ independently in the effective non-magnetic system instead of only tuning the ratio $\mu / T$. This point is important when considering the thermodynamics.

- The discrete momentum $k_{\text {eff }}=\sqrt{2|q h| l}$ must be held fixed in the transformation (22). The bulk-boundary relation is particularly simple in this case, as the Landau levels can readily be seen in the bulk solution, only to remain identical in the boundary theory.

- Similar to the non-magnetic system [11], the IR physics is controlled by the near horizon $A d S_{2} \times \mathbb{R}^{2}$ geometry, which indicates the existence of an IR CFT, characterized by operators $\mathcal{O}_{l}, l \in N$ with operator dimensions $\delta=1 / 2+\nu_{l}$ :

$$
\nu_{l}=\frac{1}{6} \sqrt{6\left(m^{2}+\frac{2|q h| l}{r_{* *}^{2}}\right)-\frac{\mu_{q}^{2}}{r_{* *}^{4}}},
$$

in dimensionless notation, and $\mu_{q} \equiv \mu q$. At $T=0$, when $r_{* *}=1$, it becomes

$$
\nu_{l}=\frac{1}{6} \sqrt{6\left(m^{2}+2|q h| l\right)-\mu_{q}^{2}} \text {. }
$$

The Green's function for these operators $\mathcal{O}_{l}$ is found to be $\mathcal{G}_{l}^{R}(\omega) \sim \omega^{2 \nu_{l}}$ and the exponents $\nu_{l}$ determines the dispersion properties of the quasiparticle excitations. For $\nu>1 / 2$ the system has a stable quasiparticle and a linear dispersion, whereas for $\nu \leq 1 / 2$ one has a non-Fermi liquid with power-law dispersion and an unstable quasiparticle.

\section{MAGNETIC FIELDS AND CONFORMAL INVARIANCE}

Despite the fact that a magnetic field introduces a scale, in the absence of a chemical potential, all spectral functions are essentially still determined by conformal symmetry. To show this, we need to establish certain properties of the near-horizon geometry of a Reissner-Nordström black hole. This leads to the $A d S_{2}$ perspective that was developed in [7]. The result relies on the conformal algebra and its relation to the magnetic group, from the viewpoint of the infrared CFT that was studied in [7]. Later on we will see that the insensitivity to the magnetic field also carries over to $A d S_{4}$ and the UV CFT in some respects. To simplify the derivations, we consider the case $T=0$.

\section{A. The near-horizon limit and Dirac equation in $A d S_{2}$}

It was established in [7] that an electrically charged extremal $A d S$-Reissner-Nordström black hole has an $A d S_{2}$ throat in the inner bulk region. This conclusion carries over to the magnetic case with some minor differences. We will now give a quick derivation of the $A d S_{2}$ formalism for a dyonic black hole, referring the reader to [7] for more details (that remain largely unchanged in the magnetic field).

Near the horizon $r=r_{* *}$ of the black hole described by the metric (2), the redshift factor $f(r)$ develops a double zero:

$$
f(r)=6 \frac{\left(r-r_{* *}\right)^{2}}{r_{* *}^{2}}+\mathcal{O}\left(\left(r-r_{* *}\right)^{3}\right)
$$

Now consider the scaling limit

$$
r-r_{* *}=\lambda \frac{R_{2}^{2}}{\zeta}, \quad t=\lambda^{-1} \tau, \lambda \rightarrow 0 \text { with } \tau, \zeta \text { finite. }
$$

In this limit, the metric (2) and the gauge field reduce to

$$
\begin{aligned}
d s^{2} & =\frac{R_{2}^{2}}{\zeta^{2}}\left(-d \tau^{2}+d \zeta^{2}\right)+\frac{r_{* *}^{2}}{R^{2}}\left(d x^{2}+d y^{2}\right) \\
A_{\tau} & =\frac{\mu R_{2}^{2} r_{0}}{r_{* *}^{2}} \frac{1}{\zeta}, \quad A_{x}=H x
\end{aligned}
$$


where $R_{2}=\frac{R}{\sqrt{6}}$. The geometry described by this metric is indeed $A d S_{2} \times \mathbb{R}^{2}$. Physically, the scaling limit given in eq. (34) with finite $\tau$ corresponds to the long time limit of the original time coordinate $t$, which translates to the low frequency limit of the boundary theory:

$$
\frac{\omega}{\mu} \rightarrow 0
$$

where $\omega$ is the frequency conjugate to $t$. (One can think of $\lambda$ as being the frequency $\omega$ ). Near the $A d S_{4}$ horizon, we expect the $A d S_{2}$ region of an extremal dyonic black hole to have a $\mathrm{CFT}_{1}$ dual. We refer to [7] for an account of this $A d S_{2} / \mathrm{CFT}_{1}$ duality. The horizon of $A d S_{2}$ region is at $\zeta \rightarrow \infty$ (coefficient in front of $d \tau$ vanishes at the horizon in eq. (35) ) and the infrared CFT (IR CFT) lives at the $A d S_{2}$ boundary at $\zeta=0$. The scaling picture given by eqs. (34 35) suggests that in the low frequency limit, the 2-dimensional boundary theory is described by this IR CFT (which is a $\mathrm{CFT}_{1}$ ). The Green's function for the operator $\mathcal{O}$ in the boundary theory is obtained through a small frequency expansion and a matching procedure between the two different regions (inner and outer) along the radial direction, and can be expressed through the Green's function of the IR CFT [7].

The explicit form for the Dirac equation (A28) in the magnetic field is of little interest for the analytical results that follow; for completeness, we give it in the Appendix. Of primary interest is its limit in the IR region with metric given by eq. (35):

$$
\left(-\frac{1}{\sqrt{g_{\zeta \zeta}}} \sigma^{3} \partial_{\zeta}-m+\frac{1}{\sqrt{-g_{\tau \tau}}} \sigma^{1}\left(\omega+\frac{\mu_{q} R_{2}^{2} r_{0}}{r_{* *}^{2} \zeta}\right)-\frac{1}{\sqrt{g_{i i}} i \sigma^{2} \lambda_{l}}\right) F^{(l)}=0
$$

where the effective momentum of the $l$-th Landau level is $\lambda_{l}=\sqrt{2|q h| l}, \mu_{q} \equiv \mu q$ and we omit the index of the spinor field. To obtain eq. (37), it is convenient to pick the gamma matrix basis as $\Gamma^{\hat{\zeta}}=-\sigma_{3}, \Gamma^{\hat{\tau}}=i \sigma_{1}$ and $\Gamma^{\hat{i}}=-\sigma_{2}$. We can write explicitly:

$$
\left(\begin{array}{cc}
\frac{\zeta}{R_{2}} \partial_{\zeta}+m & -\frac{\zeta}{R_{2}}\left(\omega+\frac{\mu_{q} R_{2}^{2} r_{0}}{r_{* *}^{2} \zeta}\right)+\frac{R}{r_{* *}} \lambda_{l} \\
\frac{\zeta}{R_{2}}\left(\omega+\frac{\mu_{q} R_{2}^{2} r_{0}}{r_{* *}^{2} \zeta}\right)+\frac{R}{r_{* *}} \lambda_{l} & \frac{\zeta}{R_{2}} \partial_{\zeta}-m
\end{array}\right)\left(\begin{array}{l}
y \\
z
\end{array}\right)=0 .
$$

Note that the $A d S_{2}$ radius $R_{2}$ enters for the $(\tau, \zeta)$ directions. At the $A d S_{2}$ boundary, $\zeta \rightarrow 0$, the Dirac equation to the leading order is given by

$$
\zeta \partial_{\zeta} F^{(l)}=-U F^{(l)}, \quad U=R_{2}\left(\begin{array}{cc}
m & -\frac{\mu_{q} R_{2} r_{0}}{r_{* *}^{2}}+\frac{R}{r_{* *}} \lambda_{l} \\
\frac{\mu_{q} R_{2} r_{0}}{r_{* *}^{2}}+\frac{R}{r_{* *}} \lambda_{l} & -m
\end{array}\right)
$$

The solution to this equation is given by the scaling function $F^{(l)}=A e_{+} \zeta^{-\nu_{l}}+B e_{-} \zeta^{\nu_{l}}$ where $e_{ \pm}$are the real eigenvectors of $U$ and the exponent is

$$
\nu_{l}=\frac{1}{6} \sqrt{6\left(m^{2}+\frac{R^{2}}{r_{* *}^{2}} 2|q h| l\right) R^{2}-\frac{\mu_{q}^{2} R^{4} r_{0}^{2}}{r_{* *}^{4}}}
$$

The conformal dimension of the operator $\mathcal{O}$ in the IR CFT is $\delta_{l}=\frac{1}{2}+\nu_{l}$. Comparing eq. (40) to the expression for the scaling exponent in [7], we conclude that the scaling properties and the $A d S_{2}$ construction are unmodified by the magnetic field, except that the scaling exponents are now fixed by the Landau quantization. This "quantization rule" was already exploited in [22] to study de Haas-van Alphen oscillations.

\section{SPECTRAL FUNCTIONS}

In this section we will explore some of the properties of the spectral function, in both plane wave and Landau level basis. We first consider some characteristic cases in the plane wave basis and make connection with the ARPES measurements. 


\section{A. Relating to the ARPES measurements}

In reality, ARPES measurements cannot be performed in magnetic fields so the holographic approach, allowing a direct insight into the propagator structure and the spectral function, is especially helpful. This follows from the observation that the spectral functions as measured in ARPES are always expressed in the plane wave basis of the photon, thus in a magnetic field, when the momentum is not a good quantum number anymore, it becomes impossible to perform the photoemission spectroscopy.

In order to compute the spectral function, we have to choose a particular fermionic plane wave as a probe. Since the separation of variables is valid throughout the bulk, the basis transformation can be performed at every constant $r$-slice. This means that only the $x$ and $y$ coordinates have to be taken into account (the plane wave probe lives only at the CFT side of the duality). We take a plane wave propagating in the $+x$ direction with spin up along the $r$-axis. In its rest frame such a particle can be described by

$$
\Psi_{\text {probe }}=e^{i \omega t-i p_{x} x}\left(\begin{array}{l}
\xi \\
\xi
\end{array}\right), \quad \xi=\left(\begin{array}{l}
1 \\
0
\end{array}\right) .
$$

Near the boundary (at $r_{b} \rightarrow \infty$ ) we can rescale our solutions of the Dirac equation making use of eqs. A23,A24, B1):

$$
F_{l}=\left(\begin{array}{c}
\zeta_{l}^{(1)}(\tilde{x}) \\
\xi_{+}^{(l)}\left(r_{b}\right) \zeta_{l}^{(1)}(\tilde{x}) \\
\zeta_{l}^{(2)}(\tilde{x}) \\
-\xi_{+}^{(l)}\left(r_{b}\right) \zeta_{l}^{(2)}(\tilde{x})
\end{array}\right), \quad \tilde{F}_{l}=\left(\begin{array}{c}
\zeta_{l}^{(1)}(\tilde{x}) \\
\xi_{-}^{(l)}\left(r_{b}\right) \zeta_{l}^{(1)}(\tilde{x}) \\
-\zeta_{l}^{(2)}(\tilde{x}) \\
\xi_{-}^{(l)}\left(r_{b}\right) \zeta_{l}^{(2)}(\tilde{x})
\end{array}\right)
$$

with rescaled $\tilde{x}$ defined after eq. (A20). This representation is useful since we calculate the components $\xi_{ \pm}\left(r_{b}\right)$ related to the retarded Green's function in our numerics (we keep the notation of [7]).

Let $\mathcal{O}_{l}$ and $\tilde{\mathcal{O}}_{l}$ be the CFT operators dual to respectively $F_{l}$ and $\tilde{F}_{l}$, and $c_{k}^{\dagger}, c_{k}$ be the creation and annihilation

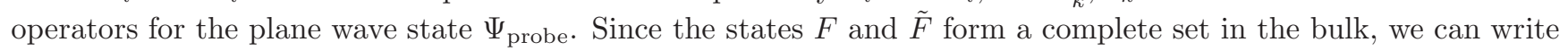

$$
c_{p}^{\dagger}(\omega)=\sum_{l}\left(U_{l}^{*}, \tilde{U}_{l}^{*}\right)\left(\begin{array}{c}
\mathcal{O}_{l}^{\dagger}(\omega) \\
\tilde{\mathcal{O}}_{l}^{\dagger}(\omega)
\end{array}\right)=\sum_{l}\left(U_{l}^{*} \mathcal{O}_{l}^{\dagger}(\omega)+\tilde{U}_{l}^{*} \tilde{\mathcal{O}}_{l}^{\dagger}(\omega)\right)
$$

where the overlap coefficients $U_{l}(\omega)$ are given by the inner product between $\Psi_{\text {probe }}$ and $F$ :

$$
U_{l}\left(p_{x}\right)=\int d x F_{l}^{\dagger} i \Gamma^{0} \Psi_{\text {probe }}=-\int d x e^{-i p_{x} x} \xi_{+}\left(r_{b}\right)\left(\zeta_{l}^{(1) \dagger}(\tilde{x})-\zeta_{l}^{(2) \dagger}(\tilde{x})\right)
$$

with $\bar{F}=F^{\dagger} i \Gamma^{0}$, and similar expression for $\tilde{U}_{l}$ involving $\xi_{-}\left(r_{b}\right)$. The constants $U_{l}$ can be calculated analytically using the numerical value of $\xi_{ \pm}\left(r_{b}\right)$, and by noting that the Hermite functions are eigenfunctions of the Fourier transform. We are interested in the retarded Green's function, defined as

$$
\begin{aligned}
G_{\mathcal{O}_{l}}^{R}(\omega, p) & =-i \int d^{x} d t e^{i \omega t-i p \cdot x} \theta(t) G_{\mathcal{O}_{l}}^{R}(t, x) \\
G_{\mathcal{O}_{l}}^{R}(t, x) & =\left\langle 0\left|\left[\mathcal{O}_{l}(t, x), \overline{\mathcal{O}}_{l}(0,0)\right]\right| 0\right\rangle \\
G^{R} & =\left(\begin{array}{cc}
G_{\mathcal{O}} & 0 \\
0 & \tilde{G}_{\mathcal{O}}
\end{array}\right),
\end{aligned}
$$

where $\tilde{G}_{\mathcal{O}}$ is the retarded Green's function for the operator $\tilde{\mathcal{O}}$.

Exploiting the orthogonality of the spinors created by $\mathcal{O}$ and $\mathcal{O}^{\dagger}$ and using eq. (43), the Green's function in the plane wave basis can be written as

$$
G_{c_{p}}^{R}\left(\omega, p_{x}\right)=\sum_{l} \operatorname{tr}\left(\begin{array}{c}
U \\
\tilde{U}
\end{array}\right)\left(U^{*}, \tilde{U}^{*}\right) G^{R}=\left(\left|U_{l}\left(p_{x}\right)\right|^{2} G_{\mathcal{O}_{l}}^{R}(\omega, l)+\left|\tilde{U}_{l}\left(p_{x}\right)\right|^{2} \tilde{G}_{\mathcal{O}_{l}}^{R}(\omega, l)\right)
$$

In practice, we cannot perform the sum in eq. (46) all the way to infinity, so we have to introduce a cutoff Landau level $l_{\text {cut }}$. In most cases we are able to make $l_{\text {cut }}$ large enough that the behavior of the spectral function is clear. 
Using the above formalism, we have produced spectral functions for two different conformal dimensions and fixed chemical potential and magnetic field (Fig. 1). Using the plane wave basis allows us to directly detect the Landau levels. The unit used for plotting the spectra (here and later on in the paper) is the effective temperature $T_{\text {eff }}[5]$ :

$$
T_{\mathrm{eff}}=\frac{T}{2}\left(1+\sqrt{1+\frac{3 \mu^{2}}{(4 \pi T)^{2}}}\right) .
$$

This unit interpolates between $\mu$ at $T / \mu=0$ and $T$ and is of or $T / \mu \rightarrow \infty$, and is convenient for the reason that the relevant quantities (e. g., Fermi momentum) are of order unity for any value of $\mu$ and $h$.
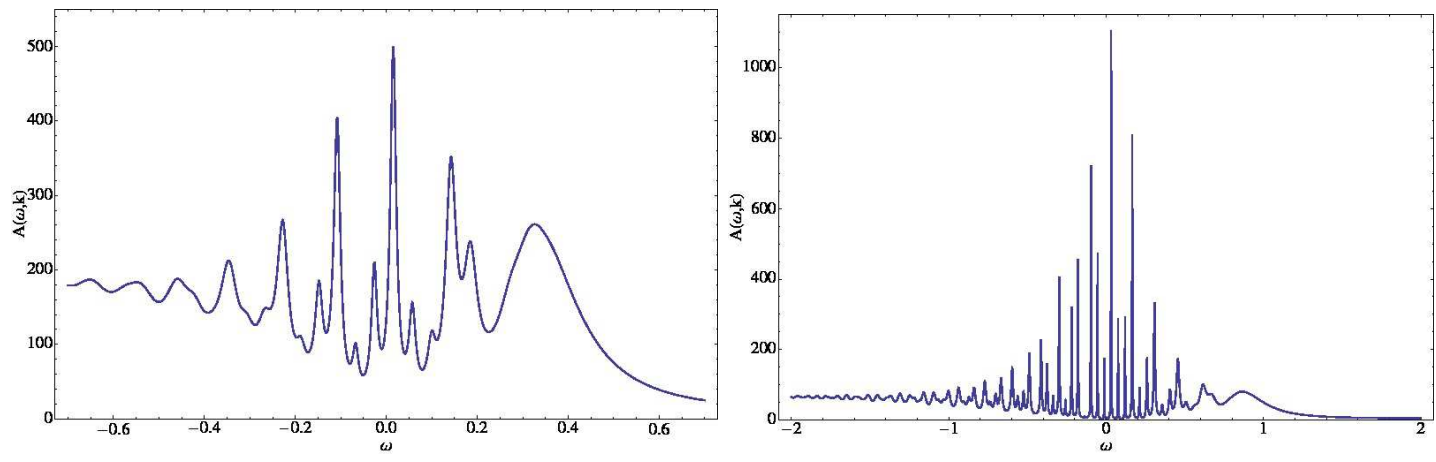

FIG. 1: Two examples of spectral functions in the plane wave basis for $\mu / T=50$ and $h / T=1$. The conformal dimension is $\Delta=5 / 4$ (left) and $\Delta=3 / 2$ (right). Frequency is in the units of effective temperature $T_{\text {eff }}$. The plane wave momentum is chosen to be $k=1$. Despite the convolution of many Landau levels, the presence of the discrete levels is obvious.

\section{B. Magnetic crossover and disappearance of the quasiparticles}

Theoretically, it is more convenient to consider the spectral functions in the Landau level basis. For definiteness let us pick a fixed conformal dimension $\Delta=\frac{5}{4}$ which corresponds to $m=-\frac{1}{4}$. In the limit of weak magnetic fields, $h / T \rightarrow 0$, we should reproduce the results that were found in [5].

In Fig. (2.A) we indeed see that the spectral function, corresponding to a low value of $\mu / T$, behaves as expected for a nearly conformal system. The spectral function is approximately symmetric about $\omega=0$, it vanishes for $|\omega|<k$, up to a small residual tail due to finite temperature, and for $|\omega| \gg k$ it scales as $\omega^{2 m}$.

In Fig. (2.B), which corresponds to a high value of $\mu / T$, we see the emergence of a sharp quasiparticle peak. This peak becomes the sharpest when the Landau level $l$ corresponding to an effective momentum $k_{\text {eff }}=\sqrt{2|q h| l}$ coincides

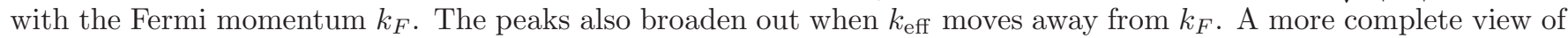
the Landau quantization in the quasiparticle regime is given in Fig. IVB, where we plot the dispersion relation $(\omega-k$ map). Both the sharp peaks and the Landau levels can be visually identified.

Collectively, the spectra in Fig. 2 show that conformality is only broken by the chemical potential $\mu$ and not by the magnetic field. Naively, the magnetic field introduces a new scale in the system. However, this scale is absent from the spectral functions, visually validating the the discussion in the previous section that the scale $h$ can be removed by a rescaling of the temperature and chemical potential.

One thus concludes that there is some value $h_{c}^{\prime}$ of the magnetic field, depending on $\mu / T$, such that the spectral function loses its quasiparticle peaks and displays near-conformal behavior for $h>h_{c}^{\prime}$. The nature of the transition and the underlying mechanism depends on the parameters $\left(\mu_{q}, T, \Delta\right)$. One mechanism, obvious from the rescaling in eq. (29), is the reduction of the effective coupling $q$ as $h$ increases. This will make the influence of the scalar potential $A_{0}$ negligible and push the system back toward conformality. Generically, the spectral function shows no sharp change but is more indicative of a crossover.

A more interesting phenomenon is the disappearance of coherent quasiparticles at high effective chemical potentials. For the special case $m=0$, we can go beyond numerics and study this transition analytically, combining the exact $T=0$ solution found in [21] and the mapping (30). In the next section, we will show that the transition is controlled by the change in the dispersion of the quasiparticle and corresponds to a sharp phase transition. Increasing the magnetic field leads to a decrease in phenomenological control parameter $\nu_{k_{F}}$. This can give rise to a transition to a non-Fermi liquid when $\nu_{k_{F}} \leq 1 / 2$, and finally to the conformal regime at $h=h_{c}^{\prime}$ when $\nu_{k_{F}}=0$ and the Fermi surface vanishes. 
(A)
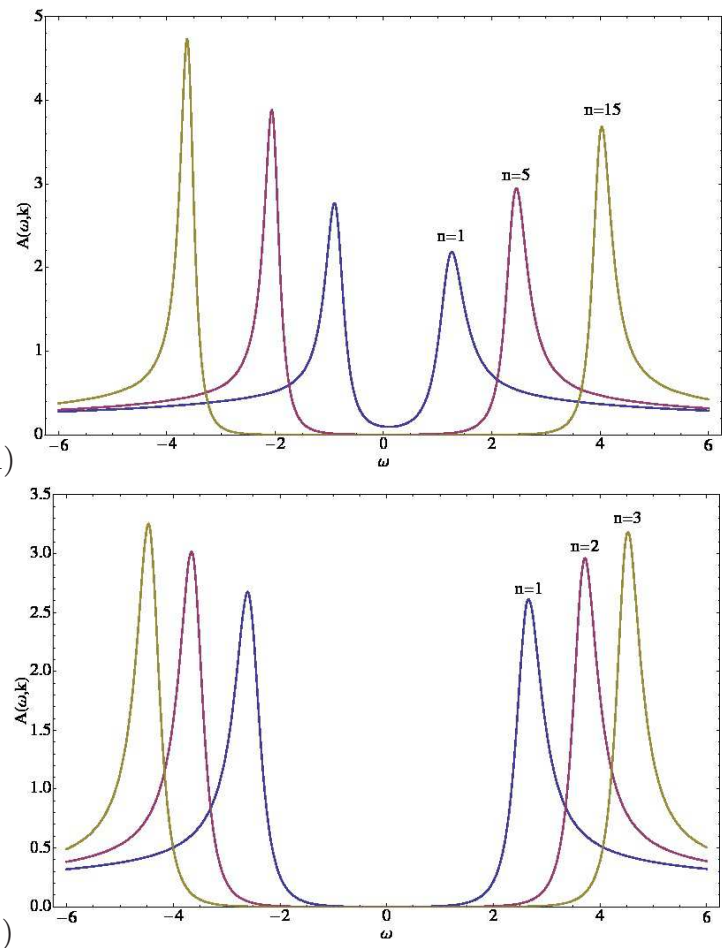

(B)

(C)

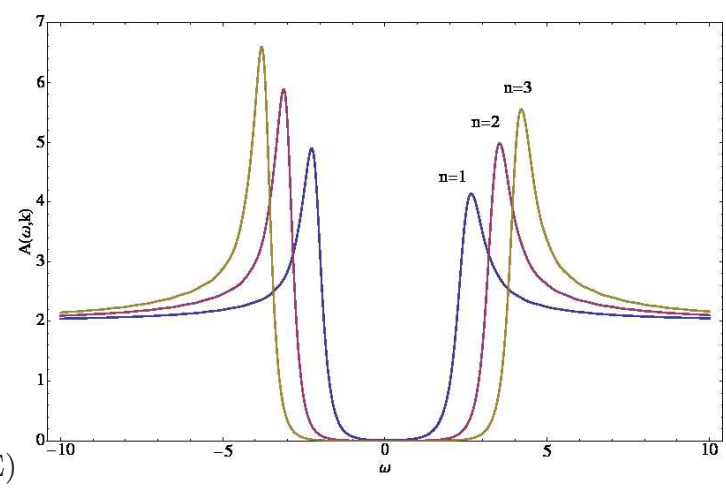

(D)
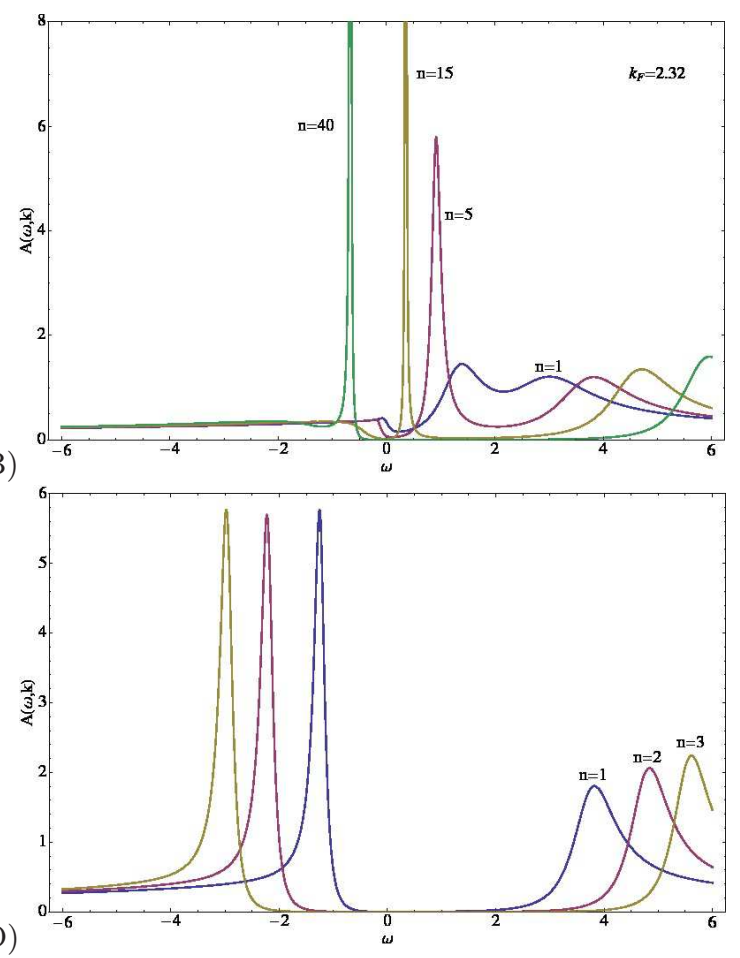

(F)
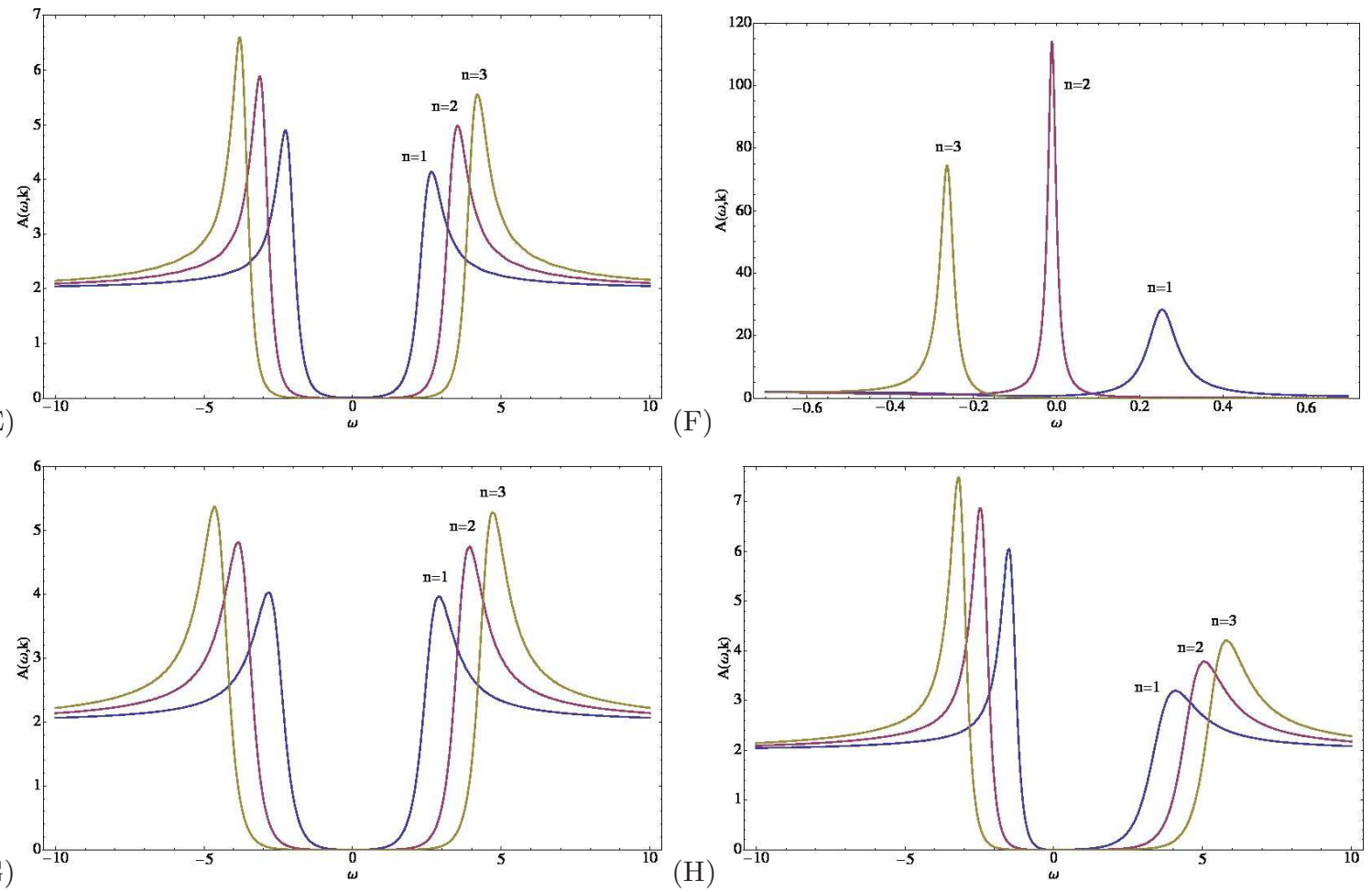

(H)

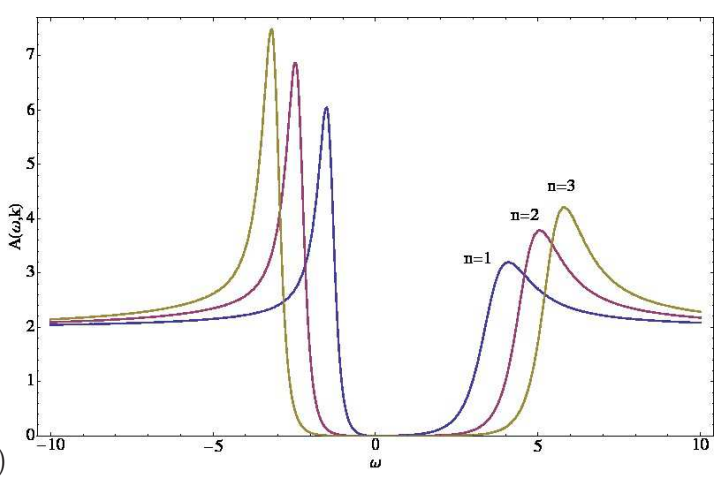

FIG. 2: Some typical examples of spectral functions $A\left(\omega, k_{\text {eff }}\right)$ vs. $\omega$ in the Landau basis, $k_{\text {eff }}=\sqrt{2|q h| n}$. The top four correspond to a conformal dimension $\Delta=\frac{5}{4} m=-\frac{1}{4}$ and the bottom four to $\Delta=\frac{3}{2}(m=0)$. In each plot we show different Landau levels, labelled by index $n$, as a function of $\mu / T$ and $h / T$. The ratios take values $(\mu / T, h / T)=$ $(1,1),(50,1),(1,50),(50,50)$ from left to right. Conformal case can be identified when $\mu / T$ is small regardless of $h / T$ (plots in the left panel). Nearly conformal behavior is seen when both $\mu / T$ and $h / T$ are large. This confirms our analytic result that the behavior of the system is primarily governed by $\mu$. Departure from the conformality and sharp quasiparticle peaks are seen when $\mu / T$ is large and $h / T$ is small in (2.B) and (2.F). Multiple quasiparticle peaks arise whenever $k_{\text {eff }}=k_{F}$. This suggests the existence of a critical magnetic field, beyond which the quasiparticle description becomes invalid and the system exhibits a conformal-like behavior. As before, the frequency $\omega$ is in units of $T_{\text {eff }}$. 

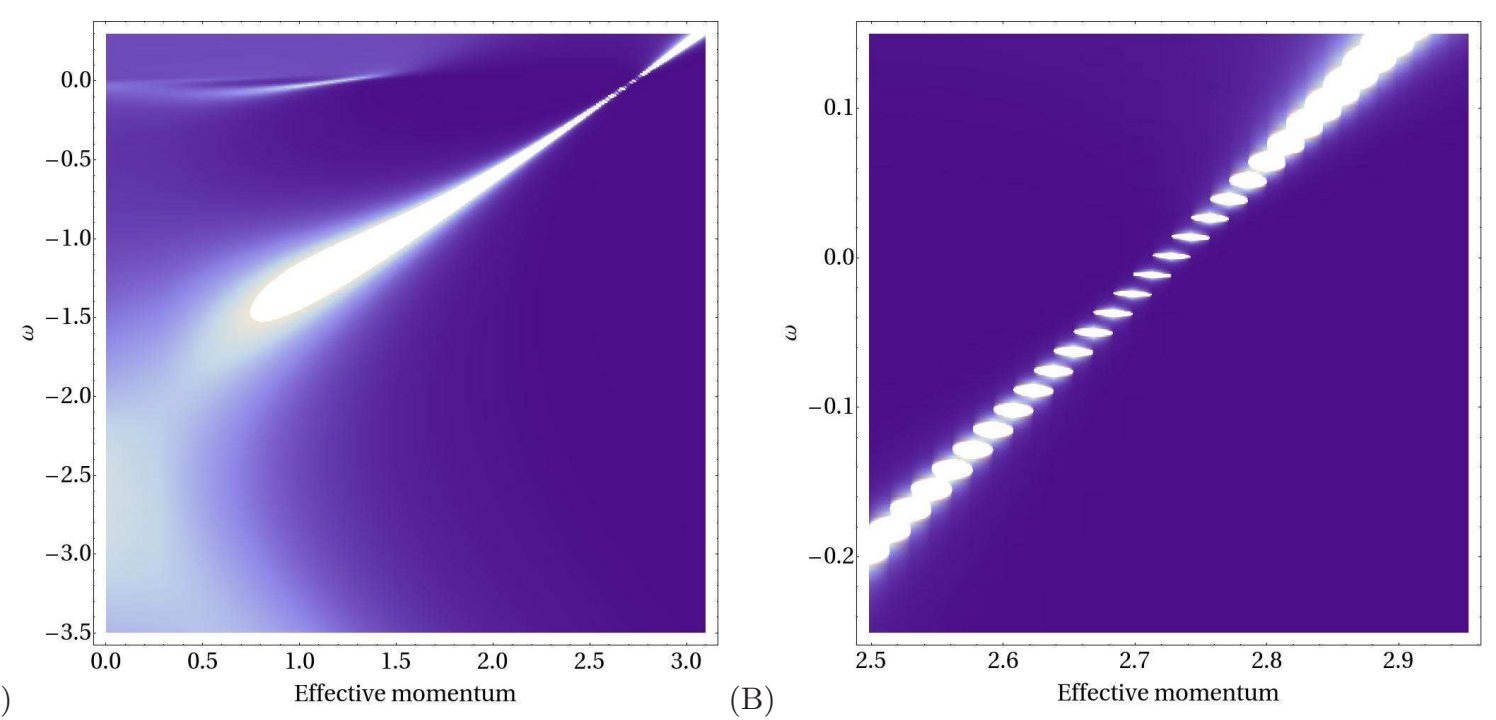

FIG. 3: Dispersion relation $\omega$ vs. $k_{\text {eff }}$ for $\mu / T=50, h / T=1$ and $\Delta=\frac{5}{4}\left(m=-\frac{1}{4}\right)$. The spectral function $A\left(\omega, k_{\text {eff }}\right)$ is displayed as a density plot. (A) On a large energy and momentum scale, we clearly sees that the peaks disperse almost linearly $\left(\omega \approx v_{F} k\right)$, indicating that we are in the stable quasiparticle regime. (B) A zoom-in near the location of the Fermi surface shows clear Landau quantization.

(A)

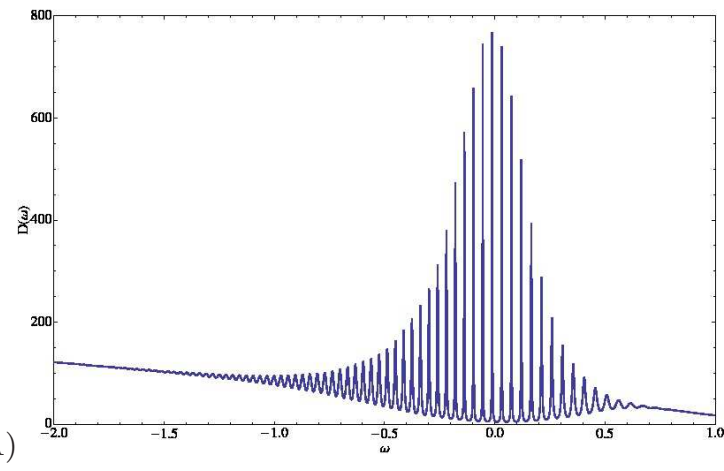

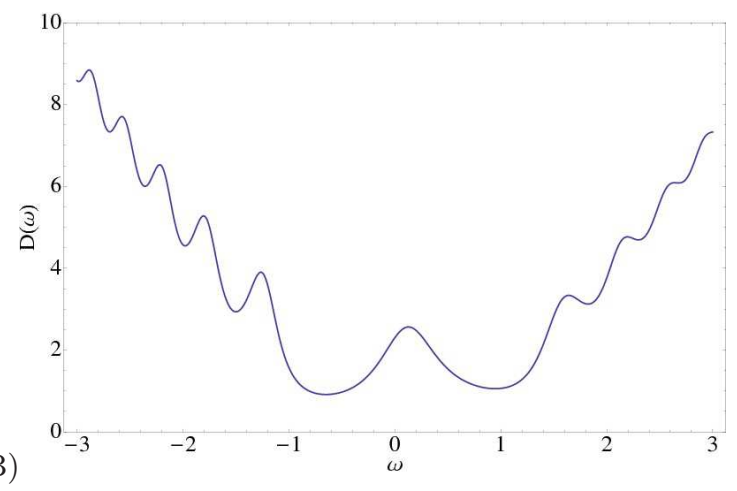

(B)

FIG. 4: Density of states $D(\omega)$ for $m=-\frac{1}{4}$ and (A) $\mu / T=50, h / T=1$, and (B) $\mu / T=1, h / T=1$. Sharp quasiparticle peaks from the splitting of the Fermi surface are clearly visible in (A). The case (B) shows square-root level spacing characteristic of a (nearly) Lorentz invariant spectrum such as that of graphene.

\section{Density of states}

As argued at the beginning of this section, the spectral function can look quite different depending on the particular basis chosen. Though the spectral function is an attractive quantity to consider due to connection with ARPES experiments, we will also direct our attention to basis-independent and manifestly gauge invariant quantities. One of them is the density of states (DOS), defined by

$$
D(\omega)=\sum_{l} A(\omega, l),
$$

where the usual integral over the momentum is replaced by a sum since only discrete values of the momentum are allowed.

In Fig. 4, we plot the density of states for two systems. We clearly see the Landau splitting of the Fermi surface. A peculiar feature of these plots is that the DOS seems to grow for negative values of $\omega$. This, however, is an artefact of our calculation. Each individual spectrum in the sum eq. (48) has a finite tail that scales as $\omega^{2 m}$ for large $\omega$, so each term has a finite contribution for large values of $\omega$. When the full sum is performed, this fact implies that 
$\lim _{\omega \rightarrow \infty} D(\omega) \rightarrow \infty$. The relevant information on the density of states can be obtained by regularizing the sum, which in practice is done by summing over a finite number of terms only, and then considering the peaks that lie on top of the resulting finite-sized envelope. The physical point in Fig. 4A is the linear spacing of Landau levels, corresponding to a non-relativistic system at finite density. This is to be contrasted with Fig. 4B where the level spacing behaves as $\propto \sqrt{h}$, appropriate for a Lorentz invariant system and realized in graphene [24].

\section{FERMI LEVEL STRUCTURE AT ZERO TEMPERATURE}

In this section, we solve the Dirac equation in the magnetic field for the special case $m=0\left(\Delta=\frac{3}{2}\right)$. Although there are no additional symmetries in this case, it is possible to get an analytic solution. Using this solution, we obtain Fermi level parameters such as $k_{F}$ and $v_{F}$ and consider the process of filling the Landau levels as the magnetic field is varied.

\section{A. Dirac equation with $m=0$}

In the case $m=0$, it is convenient to solve the Dirac equation including the spin connection (eq. A2) rather than scaling it out:

$$
\begin{aligned}
\left(-\frac{\sqrt{g_{i i}}}{\sqrt{g_{r r}}} \sigma^{1} \partial_{r}-\frac{\sqrt{g_{i i}}}{\sqrt{-g_{t t}}} \sigma^{3}\left(\omega+q A_{t}\right)+\frac{\sqrt{g_{i i}}}{\sqrt{-g_{t t}}} \sigma^{1} \frac{1}{2} \omega_{\hat{t} \hat{r} t}\right. \\
\left.-\sigma^{1} \frac{1}{2} \omega_{\hat{x} \hat{r} x}-\sigma^{1} \frac{1}{2} \omega_{\hat{y} \hat{r} y}-\lambda_{l}\right) \otimes 1\left(\begin{array}{c}
\psi_{1} \\
\psi_{2}
\end{array}\right)=0,
\end{aligned}
$$

where $\lambda_{l}=\sqrt{2|q h| l}$ are the energies of the Landau levels $l=0,1, \ldots, g_{i i} \equiv g_{x x}=g_{y y}, A_{t}(r)$ is given by eq. (3), and the gamma matrices are defined in eq. (A12). In the basis of eq. (A12), the two components $\psi_{1}$ and $\psi_{2}$ decouple. Therefore, in what follows we solve for the first component only (we omit index 1). Substituting the spin connection, we have [18]:

$$
\left(-\frac{r^{2} \sqrt{f}}{R^{2}} \sigma^{1} \partial_{r}-\frac{1}{\sqrt{f}} \sigma^{3}\left(\omega+q A_{t}\right)-\sigma^{1} \frac{r \sqrt{f}}{2 R^{2}}\left(3+\frac{r f^{\prime}}{2 f}\right)-\lambda_{l}\right) \psi=0
$$

with $\psi=\left(y_{1}, y_{2}\right)$. It is convenient to change to the basis

$$
\left(\begin{array}{c}
\tilde{y_{1}} \\
\tilde{y_{2}}
\end{array}\right)=\left(\begin{array}{cc}
1 & -i \\
-i & 1
\end{array}\right)\left(\begin{array}{l}
y_{1} \\
y_{2}
\end{array}\right)
$$

which diagonalizes the system into a second order differential equation for each component. We introduce the dimensionless variables as in eqs. (15][17), and make a change of the dimensionless radial variable:

$$
r=\frac{1}{1-z},
$$

with the horizon now being at $z=0$, and the conformal boundary at $z=1$. Performing these transformations in eq. (50), the second order differential equations for $\tilde{y}_{1}$ reads

$$
\begin{aligned}
\left(f \partial_{z}^{2}+\left(\frac{3 f}{1-z}+\right.\right. & \left.f^{\prime}\right) \partial_{z}+\frac{15 f}{4(1-z)^{2}}+\frac{3 f^{\prime}}{2(1-z)}+\frac{f^{\prime \prime}}{4} \\
& \left.+\frac{1}{f}\left((\omega+q \mu z) \pm \frac{i f^{\prime}}{4}\right)^{2}-i q \mu-\lambda_{l}^{2}\right) \tilde{y}_{1}=0
\end{aligned}
$$

The second component $\tilde{y}_{2}$ obeys the same equation with $\mu \mapsto-\mu$.

At $T=0$,

$$
f=3 z^{2}\left(z-z_{0}\right)\left(z-\bar{z}_{0}\right), \quad z_{0}=\frac{1}{3}(4+i \sqrt{2}) .
$$


The solution of this fermion system at zero magnetic field and zero temperature $T=0$ has been found in [21]. To solve eq. (53), we use the mapping to a zero magnetic field system eq. (29). The combination $\mu_{q} \equiv \mu q$ at non-zero $h$ maps to $\mu_{q, \text { eff }} \equiv \mu_{\text {eff }} q_{\text {eff }}$ at zero $h$ as follows:

$$
\mu_{q} \mapsto q \sqrt{1-\frac{H^{2}}{Q^{2}+H^{2}}} \cdot g_{F} \sqrt{Q^{2}+H^{2}}=\sqrt{3} q g_{F} \sqrt{1-\frac{H^{2}}{3}}=\mu_{q, \text { eff }}
$$

where at $T=0$ we used $Q^{2}+H^{2}=3$. We solve eq. (53) for zero modes, i. e. $\omega=0$, and at the Fermi surface $\lambda=k$, and implement eq. (55).

Near the horizon $\left(z=0, f=6 z^{2}\right)$, we have

$$
6 z^{2} \tilde{y}_{1 ; 2}^{\prime \prime}+12 z \tilde{y}_{1 ; 2}^{\prime}+\left(\frac{3}{2}+\frac{\left(\mu_{q, \mathrm{e} f f}\right)^{2}}{6}-k_{F}^{2}\right) \tilde{y}_{1 ; 2}=0
$$

which gives the following behavior:

$$
\tilde{y}_{1 ; 2} \sim z^{-\frac{1}{2} \pm \nu_{k}}
$$

with the scaling exponent $\nu$ following from eq. (32):

$$
\nu=\frac{1}{6} \sqrt{6 k^{2}-\left(\mu_{q, \mathrm{e} f f}\right)^{2}}
$$

at the momentum $k$. Using Maple, we find the zero mode solution of eq. (53) with a regular behavior $z^{-\frac{1}{2}+\nu}$ at the horizon [18, 21]:

$$
\begin{aligned}
\tilde{y}_{1}^{(0)} & =N_{1}(z-1)^{\frac{3}{2}} z^{-\frac{1}{2}+\nu}\left(z-\bar{z}_{0}\right)^{-\frac{1}{2}-\nu}\left(\frac{z-z_{0}}{z-\bar{z}_{0}}\right)^{\frac{1}{4}\left(-1-\sqrt{2} \mu_{q, \mathrm{e} f f} / z_{0}\right)} \\
& \times{ }_{2} F_{1}\left(\frac{1}{2}+\nu-\frac{\sqrt{2}}{3} \mu_{q, \mathrm{eff}}, \nu+i \frac{\mu_{q, \mathrm{e} f f}}{6}, 1+2 \nu, \frac{2 i \sqrt{2} z}{3 z_{0}\left(z-\bar{z}_{0}\right)}\right)
\end{aligned}
$$

and

$$
\begin{aligned}
\tilde{y}_{2}^{(0)} & =N_{2}(z-1)^{\frac{3}{2}} z^{-\frac{1}{2}+\nu}\left(z-\bar{z}_{0}\right)^{-\frac{1}{2}-\nu}\left(\frac{z-z_{0}}{z-\bar{z}_{0}}\right)^{\frac{1}{4}\left(-1+\sqrt{2} \mu_{q, \mathrm{e} f f} / z_{0}\right)} \\
& \times{ }_{2} F_{1}\left(\frac{1}{2}+\nu+\frac{\sqrt{2}}{3} \mu_{q, \mathrm{eff}}, \nu-i \frac{\mu_{q, \mathrm{e} f f}}{6}, 1+2 \nu, \frac{2 i \sqrt{2} z}{3 z_{0}\left(z-\bar{z}_{0}\right)}\right)
\end{aligned}
$$

where ${ }_{2} F_{1}$ is the hypergeometric function and $N_{1}, N_{2}$ are normalization factors. Since normalization factors are constants, we find their relative weight by substituting solutions given in eq. (59) back into the first order differential equations at $z \sim 0$,

$$
\frac{N_{1}}{N_{2}}=-\frac{6 i \nu+\mu_{q, \mathrm{e} f f}}{\sqrt{6} k}\left(\frac{z_{0}}{\bar{z}_{0}}\right)^{\mu_{q, \mathrm{e} f f} / \sqrt{2} z_{0}} .
$$

The same relations are obtained when calculations are done for any $z$. The second solution $\tilde{\eta}_{1 ; 2}^{(0)}$, with behavior $z^{-\frac{1}{2}-\nu}$ at the horizon, is obtained by replacing $\nu \rightarrow-\nu$ in eq. (59).

To get insight into the zero-mode solution (59), we plot the radial profile for the density function $\psi^{(0) \dagger} \psi^{(0)}$ for different magnetic fields in Fig. (5). The momentum chosen is the Fermi momentum of the first Fermi surface (see the next section). The curves are normalized to have the same maxima. Magnetic field is increased from right to left. At small magnetic field, the zero modes are supported away from the horizon, while at large magnetic field, the zero modes are supported near the horizon. This means that at large magnetic field the influence of the black hole to the Fermi level structure becomes more important. 


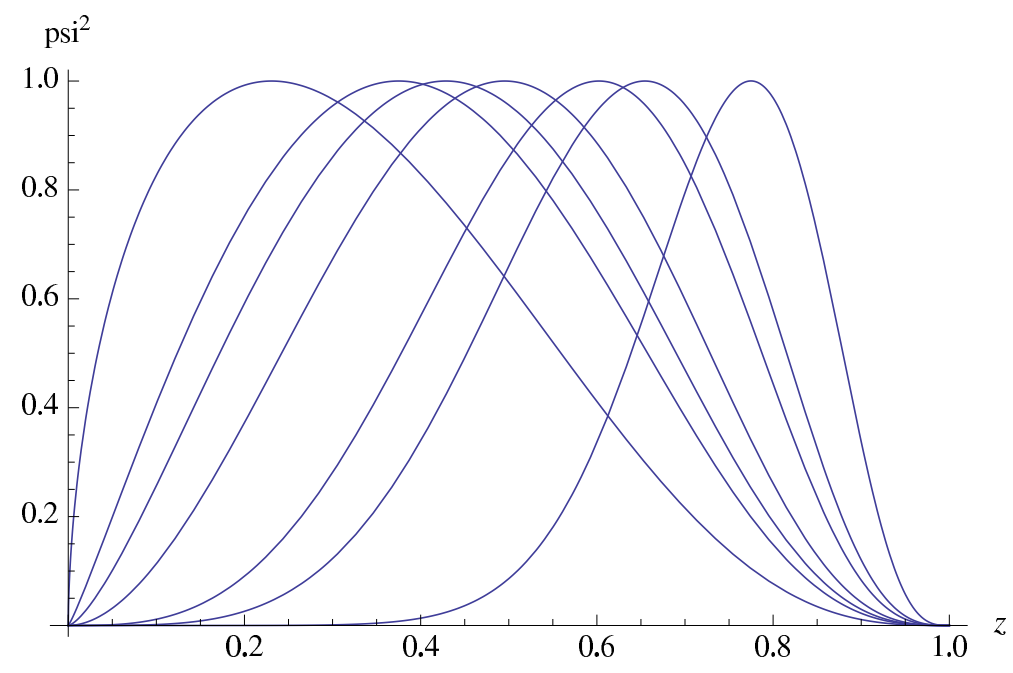

FIG. 5: Density of the zero mode $\psi^{0 \dagger} \psi^{0}$ vs. the radial coordinate $z$ (the horizon is at $z=0$ and the boundary is at $z=1$ ) for different values of the magnetic field $h$ for the first (with the largest root for $k_{F}$ ) Fermi surface. We set $g_{F}=1(h \rightarrow H)$ and $q=\frac{15}{\sqrt{3}}\left(\mu_{q, \text { eff }} \rightarrow 15 \sqrt{1-\frac{H^{2}}{3}}\right)$. From right to left the values of the magnetic field are $H=\{0,1.40,1.50,1.60,1.63,1.65,1.68\}$. The amplitudes of the curves are normalized to unity. At weak magnetic fields, the wave function is supported away from the horizon while at strong fields it is supported near the horizon.

\section{B. Magnetic effects on the Fermi momentum and Fermi velocity at $T=0$}

In the presence of a magnetic field there is only a true pole in the Green's function whenever the Landau level crosses the Fermi energy [22]

$$
2 l|q h|=k_{F}^{2} .
$$

As shown in Fig. 2, whenever the equation (62) is satisfied the spectral function $A(\omega)$ has a (sharp) peak. This is not surprising since quasiparticles can be easily excited from the Fermi surface. From eq. (62), the spectral function $A(\omega)$ and the density of states on the Fermi surface $D(\omega)$ are periodic in $\frac{1}{h}$ with the period

$$
\Delta\left(\frac{1}{h}\right)=\frac{2 \pi q}{A_{F}}
$$

where $A_{F}=\pi k_{F}^{2}$ is the area of the Fermi surface [22]. This is a manifestation of the de Haas-van Alphen quantum oscillations. At $T=0$, the electronic properties of metals depend on the density of states on the Fermi surface. Therefore, an oscillatory behavior as a function of magnetic field should appear in any quantity that depends on the density of states on the Fermi energy. Magnetic susceptibility [22] and magnetization together with the superconducting gap 23. have been shown to exhibit quantum oscillations. Every Landau level contributes an oscillating term and the period of the $l$-th level oscillation is determined by the value of the magnetic field $h$ that satisfies eq. (62) for the given value of $k_{F}$. Quantum oscillations (and the quantum Hall effect which we consider later in the paper) are examples of phenomena in which Landau level physics reveals the presence of the Fermi surface. The superconducting gap found in the quark matter in magnetic fields [23] is another evidence for the existence of the (highly degenerate) Fermi surface and the corresponding Fermi momentum.

Generally, a Fermi surface controls the occupation of energy levels in the system: the energy levels below the Fermi surface are filled and those above are empty (or non-existent). Here, however, the association to the Fermi momentum can be obscured by the fact that the fermions form highly degenerate Landau levels. Thus, in two dimensions, in the presence of the magnetic field the corresponding effective Fermi surface is given by a single point in the phase space, that is determined by $n_{F}$, the Landau index of the highest occupied level, i.e., the highest Landau level below the chemical potential ${ }^{2}$. Increasing the magnetic field, Landau levels 'move up' in the phase space leaving only the

\footnotetext{
${ }^{2}$ We would like to thank Igor Shovkovy for clarifying the issue with the Fermi momentum in the presence of the magnetic field.
} 
lower levels occupied, so that the effective Fermi momentum scales roughly (excluding interactions) as a square root of the magnetic field, $k_{F} \sim \sqrt{n_{F}} \sim k_{F}^{\max } \sqrt{1-h / h_{\max }}$. High magnetic fields drive the effective density of the charge carriers down, approaching the limit when the Fermi momentum coincides with the lowest Landau level.

Many phenomena observed in the paper can thus be qualitatively explained by Landau quantization. As discussed before, the notion of the Fermi momentum is lost at very high magnetic fields. In what follows, the quantitative Fermi level structure at zero temperature, described by $k_{F}$ and $v_{F}$ values, is obtained as a function of the magnetic field using the solution of the Dirac equation given by eqs. (59]60). As in [11], we neglect first the discrete nature of the Fermi momentum and velocity in order to obtain general understanding. Upon taking the quantization into account, the smooth curves become combinations of step functions following the same trend as the smooth curves (without quantization). While usually the grand canonical ensemble is used, where the fixed chemical potential controls the occupation of the Landau levels [26], in our setup, the Fermi momentum is allowed to change as the magnetic field is varied, while we keep track of the IR conformal dimension $\nu$.

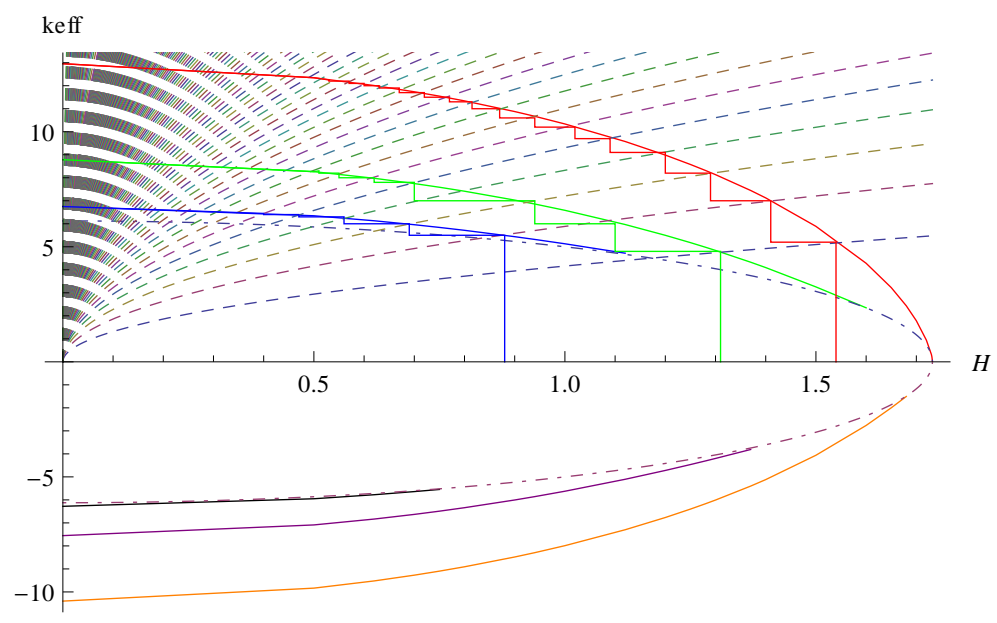

FIG. 6: Effective momentum $k_{\text {eff }}$ vs. the magnetic field $h \rightarrow H$ (we set $g_{F}=1, q=\frac{15}{\sqrt{3}}$ ). As we increase magnetic field the Fermi surface shrinks. Smooth solid curves represent situation as if momentum is a continuous parameter (for convenience), stepwise solid functions are the real Fermi momenta which are discretized due to the Landau level quantization: $k_{F} \rightarrow \sqrt{2|q h| l}$ with $l=1,2, \ldots$ where $\sqrt{2|q h| l}$ are Landau levels given by dotted lines (only positive discrete $k_{F}$ are shown). At a given $h$ there are multiple Fermi surfaces. From right to left are the first, second etc. Fermi surfaces. The dashed-dotted line is $\nu_{k_{F}}=0$ where $k_{F}$ is terminated. Positive and negative $k_{e f f}$ correspond to Fermi surfaces in two components of the Green's function.

The Fermi momentum is defined by the matching between IR and UV physics [7, therefore it is enough to know the solution at $\omega=0$, where the matching is performed. To obtain the Fermi momentum, we require that the zero mode solution is regular at the horizon $\left(\psi^{(0)} \sim z^{-\frac{1}{2}+\nu}\right)$ and normalizable at the boundary. At the boundary $z \sim 1$, the wave function behaves as

$$
a(1-z)^{\frac{3}{2}-m}\left(\begin{array}{l}
1 \\
0
\end{array}\right)+b(1-z)^{\frac{3}{2}+m}\left(\begin{array}{l}
0 \\
1
\end{array}\right) .
$$

To require it to be normalizable is to set the first term $a=0$; the wave function at $z \sim 1$ is then

$$
\psi^{(0)} \sim(1-z)^{\frac{3}{2}+m}\left(\begin{array}{l}
0 \\
1
\end{array}\right)
$$

Eq. (65) leads to the condition $\lim _{z \rightarrow 1}(z-1)^{-3 / 2}\left(\tilde{y}_{2}^{(0)}+i \tilde{y}_{1}^{(0)}\right)=0$, which, together with eq. (59), gives the following equation for the Fermi momentum as function of the magnetic field [18, 21]

$$
\frac{{ }_{2} F_{1}\left(1+\nu+\frac{i \mu_{q, \mathrm{e} f f}}{6}, \frac{1}{2}+\nu-\frac{\sqrt{2} \mu_{q, \mathrm{e} f f}}{3}, 1+2 \nu, \frac{2}{3}(1-i \sqrt{2})\right)}{{ }_{2} F_{1}\left(\nu+\frac{i \mu_{q, \mathrm{e} f f}}{6}, \frac{1}{2}+\nu-\frac{\sqrt{2} \mu_{q, \mathrm{e} f f}}{3}, 1+2 \nu, \frac{2}{3}(1-i \sqrt{2})\right)}=\frac{6 \nu-i \mu_{q, \mathrm{e} f f}}{k_{F}(-2 i+\sqrt{2})},
$$

with $\nu \equiv \nu_{k_{F}}$ given by eq. (58). Using Mathematica to evaluate the hypergeometric functions, we numerically solve the equation for the Fermi surface, which gives effective momentum as if it were continuous, i.e. when quantization 


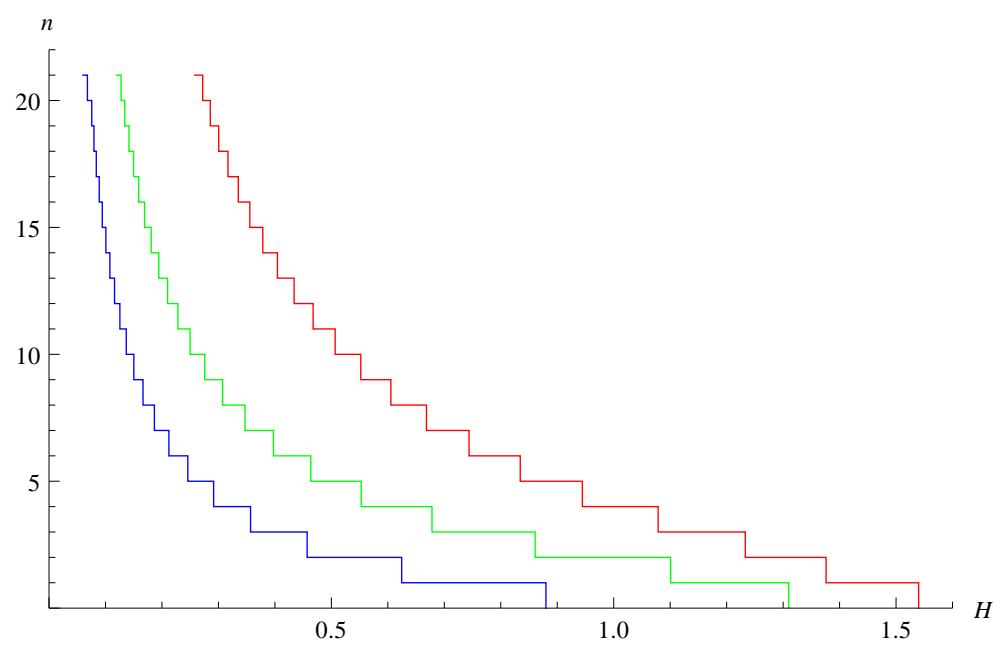

FIG. 7: Landau level numbers $n$ coresponding to the quantized Fermi momenta vs. the magnetic field $h \rightarrow H$ for the three Fermi surfaces with positive $k_{F}$. We set $g_{F}=1, q=\frac{15}{\sqrt{3}}$. From right to left are the first, second and third Fermi surfaces.

is neglected. The solutions of eq. (66) are given in Fig. (6). There are multiple Fermi surfaces for a given magnetic field $h$. Here and in all other plots we choose $g_{F}=1$, therefore $h \rightarrow H$, and $q=\frac{15}{\sqrt{3}}$. In Fig. (6), positive and negative $k_{F}$ correspond to the Fermi surfaces in the Green's functions $G_{1}$ and $G_{2}$. The relation between two components is $G_{2}(\omega, k)=G_{1}(\omega,-k)$ [6], therefore Fig. (6) is not symmetric with respect to the x-axis. Effective momenta terminate at the dashed line $\nu_{k_{F}}=0$. Taking into account Landau quantization of $k_{F} \rightarrow \sqrt{2|q h| l}$ with $l=1,2 \ldots$, the plot consists of stepwise functions tracing the existing curves (we depict only positive $k_{F}$ ). Indeed Landau quantiaztion can be also seen from the dispersion relation at Fig. (3), where only discrete values of effective momentum are allowed and the Fermi surface has been chopped up as a result of it Fig. (3B).

Our findings agree with the results for the (largest) Fermi momentum in a three-dimensional magnetic system considered in [27], compare the stepwise dependence $k_{F}(h)$ with Fig.(5) in [27].

In Fig.(7), the Landau level index $l$ is obtained from $k_{F}(h)=\sqrt{2|q h| l}$ where $k_{F}(h)$ is a numerical solution of eq. (66). Only those Landau levels which are below the Fermi surface are filled. In Fig.(6), as we decrease magnetic field first nothing happens until the next Landau level crosses the Fermi surface which corresponds to a jump up to the next step. Therefore, at strong magnetic fields, fewer states contribute to transport properties and the lowest Landau level becomes more important (see the next section). At weak magnetic fields, the sum over many Landau levels has to be taken, ending with the continuous limit as $h \rightarrow 0$, when quantization can be ignored.

In Fig. (8), we show the IR conformal dimension as a function of the magnetic field. We have used the numerical solution for $k_{F}$. Fermi liquid regime takes place at magnetic fields $h<h_{c}$, while non-Fermi liquids exist in a narrow band at $h_{c}<h<h_{c}^{\prime}$, and at $h_{c}^{\prime}$ the system becomes near-conformal.

In this figure we observe the pathway of the possible phase transition exhibited by the Fermi surface (ignoring Landau quantization): it can vanish at the line $\nu_{k_{F}}=0$, undergoing a crossover to the conformal regime, or cross the line $\nu_{k_{F}}=1 / 2$ and go through a non-Fermi liquid regime, and subsequently cross to the conformal phase. Note that the primary Fermi surface with the highest $k_{F}$ and $\nu_{k_{F}}$ seems to directly cross over to conformality, while the other Fermi surfaces first exhibit a "strange metal" phase transition. Therefore, all the Fermi momenta with $\nu_{k_{F}}>0$ contribute to the transport coefficients of the theory. In particular, at high magnetic fields when for the first (largest) Fermi surface $k_{F}^{(1)}$ is nonzero but small, the lowest Landau level $n=0$ becomes increasingly important contributing to the transport with half degeneracy factor as compared to the higher Landau levels.

In Fig. 9. we plot the Fermi momentum $k_{F}$ as a function of the magnetic field for the first Fermi surface (the largest root of eq. (66) ). Quantization is neglected here. At the left panel, the relatively small region between the dashed lines corresponds to non-Fermi liquids $0<\nu<\frac{1}{2}$. At large magnetic field, the physics of the Fermi surface is captured by the near horizon region (see also Fig. (5) which is $\mathrm{AdS}_{2} \times \mathbb{R}^{2}$. At the maximum magnetic field, $H_{\max }=\sqrt{3} \approx 1.73$, when the black hole becomes pure magnetically charged, the Fermi momentum vanishes when it crosses the line $\nu_{k_{F}}=0$. This only happens for the first Fermi surface. For the higher Fermi surfaces the Fermi momenta terminate at the line $\nu_{k_{F}}=0$, Fig. (6). Note the Fermi momentum for the first Fermi surface can be almost fully described by a function $k_{F}=k_{F}^{\max } \sqrt{1-\frac{H^{2}}{3}}$. It is tempting to view the behavior $k_{F} \sim \sqrt{H_{\max }-H}$ as a phase 

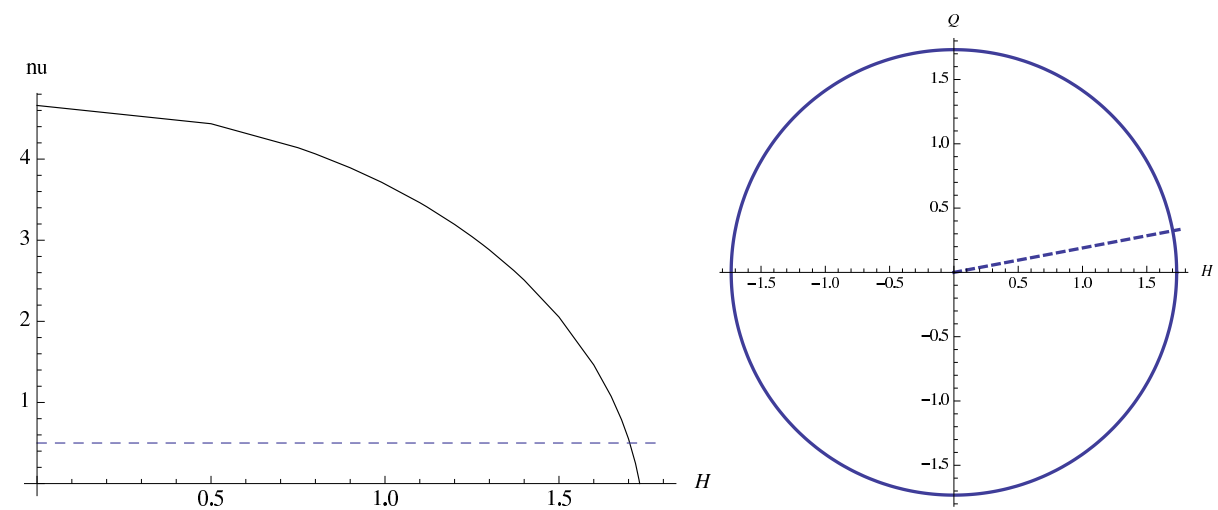

FIG. 8: Left panel. The IR conformal dimension $\nu \equiv \nu_{k_{F}}$ calculated at the Fermi momentum vs. the magnetic field $h \rightarrow H$ (we set $g_{F}=1, q=\frac{15}{\sqrt{3}}$ ). Calculations are done for the first Fermi surface. Dashed line is for $\nu=\frac{1}{2}\left(\right.$ at $H_{c}=1.70$ ), which is the border between the Fermi liquids $\nu>\frac{1}{2}$ and non-Fermi liquids $\nu<\frac{1}{2}$. Right panel. Phase diagram in terms of the chemical potential and the magnetic field $\mu^{2}+h^{2}=3$ (in dimensionless variables $h=g_{F} H, \mu=g_{F} Q$; we set $g_{F}=1$ ). Fermi liquids are above the dashed line $\left(H<H_{c}\right)$ and non-Fermi liquids are below the dashed line $\left(H>H_{c}\right)$.

transition in the system although it strictly follows from the linear scaling for $H=0$ by using the mapping (29). (Note that also $\mu=g_{F} Q=g_{F} \sqrt{3-H^{2}}$.) Taking into account the discretization of $k_{F}$, the plot will consist of an array of step functions tracing the existing curve. Our findings agree with the results for the Fermi momentum in a three dimensional magnetic system considered in [27], compare with Fig.(5) there.
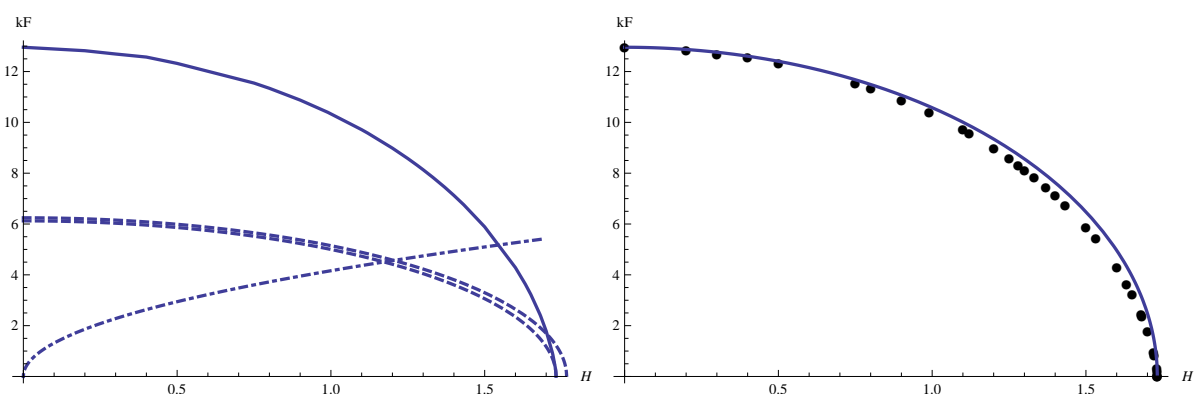

FIG. 9: Fermi momentum $k_{F}$ vs. the magnetic field $h \rightarrow H$ (we set $g_{F}=1, q=\frac{15}{\sqrt{3}}$ ) for the first Fermi surface. Left panel. The inner (closer to x-axis) dashed line is $\nu_{k_{F}}=0$ and the outer dashed line is $\nu_{k_{F}}=\frac{1}{2}$, the region between these lines corresponds to non-Fermi liquids $0<\nu_{k_{F}}<\frac{1}{2}$. The dashed-dotted line is for the first Landau level $k_{1}=\sqrt{2 q H}$. The first Fermi surface hits the border-line between a Fermi and non-Fermi liquids $\nu=\frac{1}{2}$ at $H_{c} \approx 1.70$, and it vanishes at $H_{\max }=\sqrt{3}=1.73$. Right panel. Circles are the data points for the Fermi momentum calculated analytically, solid line is a fit function $k_{F}^{\max } \sqrt{1-\frac{H^{2}}{3}}$ with $k_{F}^{\max }=12.96$.

The Fermi velocity given in eq. (27) is defined by the UV physics; therefore solutions at non-zero $\omega$ are required. The Fermi velocity is extracted from matching two solutions in the inner and outer regions at the horizon. The Fermi velocity as function of the magnetic field for $\nu>\frac{1}{2}$ is [18, 21]

$$
\begin{aligned}
v_{F} & =\frac{1}{h_{1}}\left(\int_{0}^{1} d z \sqrt{g / g_{t t}} \psi^{(0) \dagger} \psi^{(0)}\right)^{-1} \lim _{z \rightarrow 1} \frac{\left|\tilde{y}_{1}^{(0)}+i \tilde{y}_{2}^{(0)}\right|^{2}}{(1-z)^{3}}, \\
h_{1} & =\lim _{z \rightarrow 1} \frac{\tilde{y}_{1}^{(0)}+i \tilde{y}_{2}^{(0)}}{\partial_{k}\left(y_{2}^{(0)}+i \tilde{y}_{1}^{(0)}\right)},
\end{aligned}
$$

where the zero mode wavefunction is taken at $k_{F}$ eq.(159).

We plot the Fermi velocity for several Fermi surfaces in Fig. 10 and for the first Fermi surface in Fig. 111. Quantization is neglected here. The Fermi velocity is shown for $\nu>\frac{1}{2}$. It is interesting that the Fermi velocity 
vanishes when the IR conformal dimension is $\nu_{k_{F}}=\frac{1}{2}$. Formally, it follows from the fact that $v_{F} \sim(2 \nu-1)$ [7]. The first Fermi surface is at the far right. Positive and negative $v_{F}$ correspond to the Fermi surfaces in the Green's functions $G_{1}$ and $G_{2}$, respectively. The Fermi velocity $v_{F}$ has the same sign as the Fermi momentum $k_{F}$. At small magnetic field values, the Fermi velocity is very weakly dependent on $H$ and it is close to the speed of light; at large magnetic field values, the Fermi velocity rapidly decreases and vanishes (at $H_{c}=1.70$ for the first Fermi surface Fig.(11)). Geometrically, this means that with increasing magnetic field the zero mode wavefunction is supported near the black hole horizon fig.(5), where the gravitational redshift reduces the local speed of light as compared to the boundary value. It was also observed in [7, 21] at small fermion charge values.

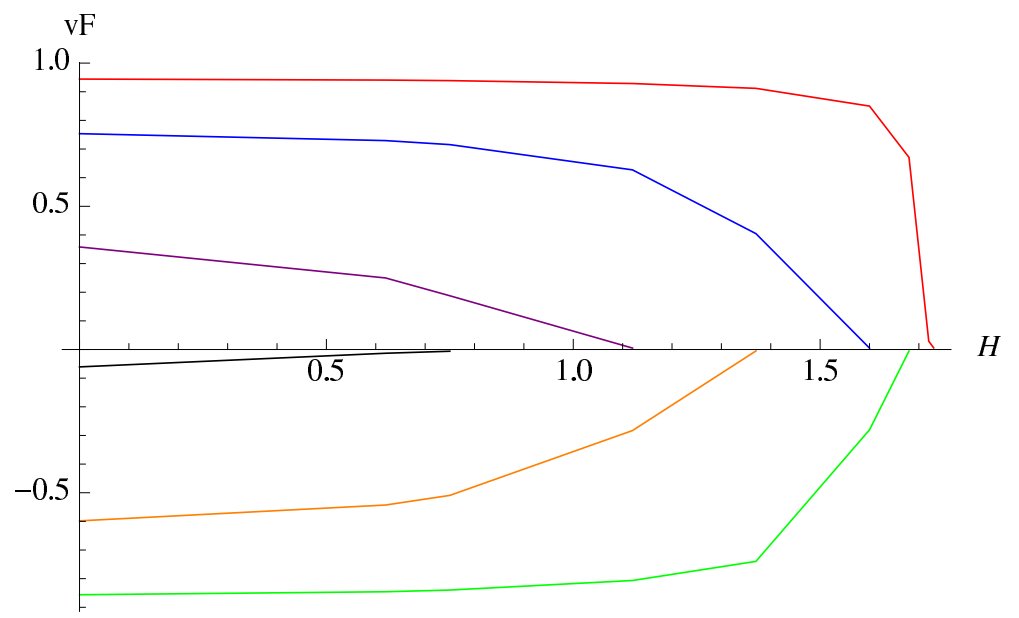

FIG. 10: Fermi velocity $v_{F}$ vs. the magnetic field $h \rightarrow H$ (we set $g_{F}=1, q=\frac{15}{\sqrt{3}}$ ) for the regime of Fermi liquids $\nu \geq \frac{1}{2}$. Fermi velocity vanishes at $\nu_{k_{F}}=\frac{1}{2}$ (x-axis). The multiple lines are for various Fermi surfaces in ascending order, with the first Fermi surface on the right. The Fermi velocity $v_{F}$ has the same sign as the Fermi momentum $k_{F}$. As above, positive and negative $v_{F}$ correspond to Fermi surfaces in the two components of the Green's function.

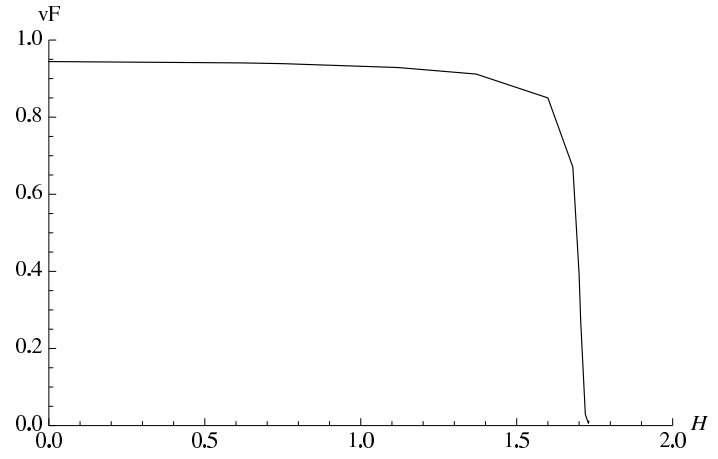

FIG. 11: Fermi velocity $v_{F}$ vs. the magnetic field $h \rightarrow H$ (we set $g_{F}=1, q=\frac{15}{\sqrt{3}}$ ) for the first Fermi surface. Fermi velocity vanishes at $\nu_{k_{F}}=\frac{1}{2}$ at $H_{c} \approx 1.70$. The region $H<H_{c}$ corresponds to the Fermi liquids and quasiparticle description.

\section{HALL AND LONGITUDINAL CONDUCTIVITIES}

In this section, we calculate the contributions to Hall $\sigma_{x y}$ and the longitudinal $\sigma_{x x}$ conductivities directly in the boundary theory. This should be contrasted with the standard holographic approach, where calculations are performed in the (bulk) gravity theory and then translated to the boundary field theory using the AdS/CFT dictionary. Specifically, the conductivity tensor has been obtained in 10 by calculating the on-shell renormalized action for the gauge field on the gravity side and using the gauge/gravity duality $A_{M} \rightarrow j_{\mu}$ to extract the $R$ charge current-current correlator at the boundary. Here, the Kubo formula involving the current-current correlator is used directly by 
utilizing the fermion Green's functions extracted from holography in 7]. Therefore, the conductivity is obtained for the charge carriers described by the fermionic operators of the boundary field theory.

The use of the conventional Kubo formulo to extract the contribution to the transport due to fermions is validated in that it also follows from a direct AdS/CFT computation of the one-loop correction to the on-shell renormalized AdS action [15]. We study in particular stable quasiparticles with $\nu>\frac{1}{2}$ and at zero temperature. This regime effectively reduces to the clean limit where the imaginary part of the self-energy vanishes $\operatorname{Im} \Sigma \rightarrow 0$. We use the gravity- "dressed" fermion propagator from eq. (27) and to make the calculations complete, the "dressed" vertex is necessary, to satisfy the Ward identities. As was argued in [15], the boundary vertex which is obtained from the bulk calculations can be approximated by a constant in the low temperature limit. Also, according to [25], the vertex only contains singularities of the product of the Green's functions. Therefore, dressing the vertex will not change the dependence of the DC conductivity on the magnetic field 25]. In addition, the zero magnetic field limit of the formulae for conductivity obtained from holography [15] and from direct boundary calculations [18] are identical.

\section{A. Integer quantum Hall effect}

Let us start from the "dressed" retarded and advanced fermion propagators [7]: $G_{R}$ is given by eq. (27) and $G_{A}=G_{R}^{*}$. To perform the Matsubara summation we use the spectral representation

$$
G\left(i \omega_{n}, \vec{k}\right)=\int \frac{d \omega}{2 \pi} \frac{A(\omega, \vec{k})}{\omega-i \omega_{n}}
$$

with the spectral function defined as $A(\omega, \vec{k})=-\frac{1}{\pi} \operatorname{Im} G_{R}(\omega, \vec{k})=\frac{1}{2 \pi i}\left(G_{R}(\omega, \vec{k})-G_{A}(\omega, \vec{k})\right)$. Generalizing to a non-zero magnetic field and spinor case [26], the spectral function [28] is

$$
A(\omega, \vec{k})=\frac{1}{\pi} \mathrm{e}^{-\frac{k^{2}}{|q h|}} \sum_{l=0}^{\infty}(-1)^{l}\left(-h_{1} v_{F}\right)\left(\frac{\Sigma_{2}\left(\omega, k_{F}\right) f(\vec{k}) \gamma^{0}}{\left(\omega+\varepsilon_{F}+\Sigma_{1}\left(\omega, k_{F}\right)-E_{l}\right)^{2}+\Sigma_{2}\left(\omega, k_{F}\right)^{2}}+\left(E_{l} \rightarrow-E_{l}\right)\right),
$$

where $\varepsilon_{F}=v_{F} k_{F}$ is the Fermi energy, $E_{l}=v_{F} \sqrt{2|q h| l}$ is the energy of the Landau level, $f(\vec{k})=P_{-} L_{l}\left(\frac{2 k^{2}}{|q h|}\right)-$ $P_{+} L_{l-1}\left(\frac{2 k^{2}}{|q h|}\right)$ with spin projection operators $P_{ \pm}=\left(1 \pm i \gamma^{1} \gamma^{2}\right) / 2$, we take $c=1$, the generalized Laguerre polynomials are $L_{n}^{\alpha}(z)$ and by definition $L_{n}(z)=L_{n}^{0}(z)$, (we omit the vector part $\vec{k} \vec{\gamma}$, it does not contribute to the DC conductivity), all $\gamma$ 's are the standard Dirac matrices, $h_{1}, v_{F}$ and $k_{F}$ are real constants (we keep the same notations for the constants as in [7]). The self-energy $\Sigma \sim \omega^{2 \nu_{k_{F}}}$ contains the real and imaginary parts, $\Sigma=\Sigma_{1}+i \Sigma_{2}$. The imaginary part comes from scattering processes of a fermion in the bulk, e. g. from pair creation, and from the scattering into the black hole. It is exactly due to inelastic/dissipative processes that we are able to obtain finite values for the transport coefficients, otherwise they are formally infinite.

Using the Kubo formula, the DC electrical conductivity tensor is

$$
\sigma_{i j}(\Omega)=\lim _{\Omega \rightarrow 0} \frac{\operatorname{Im} \Pi_{i j}^{R}}{\Omega+i 0^{+}},
$$

where $\Pi_{i j}\left(i \Omega_{m} \rightarrow \Omega+i 0^{+}\right)$is the retarded current-current correlation function; schematically the current density operator is $j^{i}(\tau, \vec{x})=q v_{F} \sum_{\sigma} \bar{\psi}_{\sigma}(\tau, \vec{x}) \gamma^{i} \psi_{\sigma}(\tau, \vec{x})$. Neglecting the vertex correction, it is given by

$$
\Pi_{i j}\left(i \Omega_{m}\right)=q^{2} v_{F}^{2} T \sum_{n=-\infty}^{\infty} \int \frac{d^{2} k}{(2 \pi)^{2}} \operatorname{tr}\left(\gamma^{i} G\left(i \omega_{n}, \vec{k}\right) \gamma^{j} G\left(i \omega_{n}+i \Omega_{m}, \vec{k}\right)\right) .
$$

The sum over the Matsubara frequency is

$$
T \sum_{n} \frac{1}{i \omega_{n}-\omega_{1}} \frac{1}{i \omega_{n}+i \Omega_{m}-\omega_{2}}=\frac{n\left(\omega_{1}\right)-n\left(\omega_{2}\right)}{i \Omega_{m}+\omega_{1}-\omega_{2}} .
$$

Taking $i \Omega_{m} \rightarrow \Omega+i 0^{+}$, the polarization operator is now

$$
\Pi_{i j}(\Omega)=\frac{d \omega_{1}}{2 \pi} \frac{d \omega_{2}}{2 \pi} \frac{n_{\mathrm{FD}}\left(\omega_{1}\right)-n_{\mathrm{FD}}\left(\omega_{2}\right)}{\Omega+\omega_{1}-\omega_{2}} \int \frac{d^{2} k}{(2 \pi)^{2}} \operatorname{tr}\left(\gamma^{i} A\left(\omega_{1}, \vec{k}\right) \gamma^{j} A\left(\omega_{2}, \vec{k}\right)\right),
$$


where the spectral function $A(\omega, \vec{k})$ is given by eq. (69) and $n_{\mathrm{FD}}(\omega)$ is the Fermi-Dirac distribution function. Evaluating the traces, we have

$$
\begin{aligned}
\sigma_{i j} & =-\frac{4 q^{2} v_{F}^{2}\left(h_{1} v_{F}\right)^{2}|q h|}{\pi \Omega} \operatorname{Re} \sum_{l, k=0}^{\infty}(-1)^{l+k+1}\left\{\delta_{i j}\left(\delta_{l, k-1}+\delta_{l-1, k}\right)+i \epsilon_{i j} \operatorname{sgn}(q h)\left(\delta_{l, k-1}-\delta_{l-1, k}\right)\right\} \\
& \times \int \frac{d \omega_{1}}{2 \pi}\left(\tanh \frac{\omega_{1}}{2 T}-\tanh \frac{\omega_{2}}{2 T}\right) \\
& \times\left(\frac{\Sigma_{2}\left(\omega_{1}\right)}{\left(\tilde{\omega}_{1}-E_{l}\right)^{2}+\Sigma_{2}^{2}\left(\omega_{1}\right)}+\left(E_{l} \rightarrow-E_{l}\right)\right)\left(\frac{\Sigma_{2}\left(\omega_{2}\right)}{\left(\tilde{\omega}_{2}-E_{k}\right)^{2}+\Sigma_{2}^{2}\left(\omega_{2}\right)}+\left(E_{k} \rightarrow-E_{k}\right)\right)
\end{aligned}
$$

with $\omega_{2}=\omega_{1}+\Omega$. We have also introduced $\tilde{\omega}_{1 ; 2} \equiv \omega_{1 ; 2}+\varepsilon_{F}+\Sigma_{1}\left(\omega_{1 ; 2}\right)$ with $\epsilon_{i j}$ being the antisymmetric tensor $\left(\epsilon_{12}=1\right)$, and $\Sigma_{1 ; 2}(\omega) \equiv \Sigma_{1 ; 2}\left(\omega, k_{F}\right)$. In the momentum integral, we use the orthogonality condition for the Laguerre polynomials $\int_{0}^{\infty} d x \mathrm{e}^{x} L_{l}(x) L_{k}(x)=\delta_{l k}$.

From eq. (74), the term symmetric/antisymmetric with respect to exchange $\omega_{1} \leftrightarrow \omega_{2}$ contributes to the diagonal/offdialgonal component of the conductivity (note the antisymmetric term $n_{\mathrm{FD}}\left(\omega_{1}\right)-n_{\mathrm{FD}}\left(\omega_{2}\right)$ ). The longitudinal and Hall DC conductivities $(\Omega \rightarrow 0)$ are thus

$$
\begin{aligned}
\sigma_{x x} & =-\frac{2 q^{2}\left(h_{1} v_{F}\right)^{2}|q h|}{\pi T} \int_{-\infty}^{\infty} \frac{d \omega}{2 \pi} \frac{\Sigma_{2}^{2}(\omega)}{\cosh ^{2} \frac{\omega}{2 T}} \\
& \times \sum_{l=0}^{\infty}\left(\frac{1}{\left(\tilde{\omega}-E_{l}\right)^{2}+\Sigma_{2}^{2}(\omega)}+\left(E_{l} \rightarrow-E_{l}\right)\right)\left(\frac{1}{\left(\tilde{\omega}-E_{l+1}\right)^{2}+\Sigma_{2}^{2}(\omega)}+\left(E_{l+1} \rightarrow-E_{l+1}\right)\right) \\
\sigma_{x y} & =-\frac{q^{2}\left(h_{1} v_{F}\right)^{2} \operatorname{sgn}(q h)}{\pi} \nu_{h} \\
\nu_{h} & =2 \int_{-\infty}^{\infty} \frac{d \omega}{2 \pi} \tanh \frac{\omega}{2 T} \Sigma_{2}(\omega) \sum_{l=0}^{\infty} \alpha_{l}\left(\frac{1}{\left(\tilde{\omega}-E_{l}\right)^{2}+\Sigma_{2}^{2}(\omega)}+\left(E_{l} \rightarrow-E_{l}\right)\right)
\end{aligned}
$$

where $\left.\tilde{\omega}=\omega+\varepsilon_{F}+\Sigma_{1}(\omega)\right)$. The filling factor $\nu_{h}$ is proportional to the density of carriers: $\left|\nu_{h}\right|=\frac{\pi}{|q h| h_{1} v_{F}} n$ (we derive this relation below eq. (89)). The degeneracy factor of the Landau levels is $\alpha_{l}: \alpha_{0}=1$ for the lowest Landau level and $\alpha_{l}=2$ for $l=1,2 \ldots$ Substituting the filling factor $\nu_{h}$ back to eq. (76), the Hall conductivity can be written as

$$
\sigma_{x y}=\frac{\rho}{h}
$$

where $\rho$ is the charge density in the boundary theory, and both the charge $q$ and the magnetic field $h$ carry a sign (the prefactor $\left(-h_{1} v_{F}\right)$ comes from the normalization choice in the fermion propagator eqs. (2769) as given in [7], which can be regarded as a factor contributing to the effective charge and is not important for further considerations). The Hall conductivity eq. (77) has been obtained using the AdS/CFT duality for the Lorentz invariant 2+ 1-dimensional boundary field theories in [10]. We recover this formula because in our case the translational invariance is maintained in the $x$ and $y$ directions of the boundary theory.

Low frequencies give the main contribution in the integrand of eq. $\Sigma_{2}(\omega) \sim \omega^{2 \nu}$ and we consider the regime $\nu>\frac{1}{2}$, we have $\Sigma_{1} \sim \Sigma_{2} \rightarrow 0$ at $\omega \sim 0$ (self-energy goes to zero faster than the $\omega$ term). Therefore, only the simple poles in the upper half-plane $\omega_{0}=-\varepsilon_{F} \pm E_{l}+\Sigma_{1}+i \Sigma_{2}$ contribute to the conductivity where $\Sigma_{1} \sim \Sigma_{2} \sim\left(-\varepsilon_{F} \pm E_{l}\right)^{2 \nu}$ are small. The same logic of calculation has been used in [26]. We obtain for the longitudinal and Hall conductivities

$$
\begin{aligned}
\sigma_{x x} & =\frac{2 q^{2}\left(h_{1} v_{F}\right)^{2} \Sigma_{2}}{\pi T} \times\left(\frac{1}{1+\cosh \frac{\varepsilon_{F}}{T}}+\sum_{l=1}^{\infty} 4 l \frac{1+\cosh \frac{\varepsilon_{F}}{T} \cosh \frac{E_{l}}{T}}{\left(\cosh \frac{\varepsilon_{F}}{T}+\cosh \frac{E_{l}}{T}\right)^{2}}\right) \\
\sigma_{x y} & =\frac{q^{2}\left(h_{1} v_{F}\right)^{2} \operatorname{sgn}(q h)}{\pi} \times 2\left(\tanh \frac{\varepsilon_{F}}{2 T}+\sum_{l=1}^{\infty}\left(\tanh \frac{\varepsilon_{F}+E_{l}}{2 T}+\tanh \frac{\varepsilon_{F}-E_{l}}{2 T}\right)\right),
\end{aligned}
$$

where the Fermi energy is $\varepsilon_{F}=v_{F} k_{F}$ and the energy of the Landau level is $E_{l}=v_{F} \sqrt{2|q h| l}$. Similar expressions were obtained in [26]. However, in our case the filling of the Landau levels is controlled by the magnetic field $h$ through the field-dependent Fermi energy $v_{F}(h) k_{F}(h)$ instead of the chemical potential $\mu$. 
At $T=0, \cosh \frac{\omega}{T} \rightarrow \frac{1}{2} \mathrm{e}^{\frac{\omega}{T}}$ and $\tanh \frac{\omega}{2 T}=1-2 n_{\mathrm{FD}}(\omega) \rightarrow \operatorname{sgn} \omega$. Therefore the longitudinal and Hall conductivities are

$$
\begin{aligned}
\sigma_{x x} & =\frac{2 q^{2}\left(h_{1} v_{F}\right)^{2} \Sigma_{2}}{\pi T} \sum_{l=1}^{\infty} l \delta_{\varepsilon_{F}, E_{l}}=\frac{2 q^{2}\left(h_{1} v_{F}\right)^{2} \Sigma_{2}}{\pi T} \times n \delta_{\varepsilon_{F}, E_{n}}, \\
\sigma_{x y} & =\frac{q^{2}\left(h_{1} v_{F}\right)^{2} \operatorname{sgn}(q h)}{\pi} 2\left(1+2 \sum_{l=1}^{\infty} \theta\left(\varepsilon_{F}-E_{l}\right)\right) \\
& =\frac{q^{2}\left(h_{1} v_{F}\right)^{2} \operatorname{sgn}(q h)}{\pi} \times 2(1+2 n) \theta\left(\varepsilon_{F}-E_{n}\right) \theta\left(E_{n+1}-\varepsilon_{F}\right),
\end{aligned}
$$

where the Landau level index runs $n=0,1, \ldots$ It can be estimated as $n=\left[\frac{k_{F}^{2}}{2|q h|}\right]$ when $v_{F} \neq 0$ ([ ] denotes the integer part), with the average spacing between the Landau levels given by the Landau energy $v_{F} \sqrt{2|q h|}$. Note that $\varepsilon_{F} \equiv \varepsilon_{F}(h)$. We can see that eq. (81) expresses the integer quantum Hall effect (IQHE). At zero temperature, as we dial the magnetic field, the Hall conductivity jumps from one quantized level to another, forming plateaus given by the filling factor

$$
\nu_{h}= \pm 2(1+2 n)= \pm 4\left(n+\frac{1}{2}\right)
$$

with $n=0,1, \ldots$ (Compare to the conventional Hall quantization $\nu_{h}= \pm 4 n$, that appears in thick graphene). Plateaus of the Hall conductivity at $T=0$ follow from the stepwise behavior of the charge density $\rho$ in eq.(77):

$$
\rho \sim 4\left(n+\frac{1}{2}\right) \theta\left(\varepsilon_{F}-E_{n}\right) \theta\left(E_{n+1}-\varepsilon_{F}\right),
$$

where $n$ Landau levels are filled and contribute to $\rho$. The longitudinal conductivity vanishes except precisely at the transition point between the plateaus. In Fig. 12, we plot the longitudinal and Hall conductivities at $T=0$, using only the terms after $\times$ sign in eq. (79). In the Hall conductivity, plateau transition occurs when the Fermi level (in Fig. 12) of the first Fermi surface $\varepsilon_{F}=v_{F}(h) k_{F}(h)$ (Figs. 911) crosses the Landau level energy as we vary the magnetic field. By decreasing the magnetic field, the plateaus become shorter and increasingly more Landau levels contribute to the Hall conductivity. This happens because of two factors: the Fermi level moves up and the spacing between the Landau levels becomes smaller. This picture does not depend on the Fermi velocity as long as it is nonzero.

In the boundary field theory, we express the charge density of the carriers (difference between the densities of "electrons" and "holes") through the Fermi energy $\varepsilon_{F}$ :

$$
n=\operatorname{tr}\left(\gamma^{0} \tilde{G}(\tau, \mathbf{0})\right), \quad \tau \rightarrow 0,
$$

where $\tilde{G}(\tau, \vec{x})$ is the translation-invariant part of the Green's function $G(\tau, \vec{x})$ from eq. (68) [26]. Using the spectral function representation eq. (69), the charge density reads

$$
n=T \sum_{n=-\infty}^{\infty} \int \frac{d^{2} k}{(2 \pi)^{2}} \int_{-\infty}^{\infty} \frac{d \omega}{2 \pi} \frac{\operatorname{tr}\left(\gamma^{0} A(\omega, \vec{k})\right)}{\omega-i \omega_{n}} .
$$

We express the Matsubara sum in terms of the contour integral over real frequencies:

$$
T \sum_{n=-\infty}^{\infty} F\left(i \omega_{n}\right) \rightarrow-\frac{i}{4 \pi} \int_{C} d z \tanh \frac{z}{2 T} F(z),
$$

where $C$ runs anti-clockwise and encircles the poles of tanh along the upper and lower half imaginary axis. We have for the charge density

$$
n=\frac{1}{2} \int \frac{d^{2} k}{(2 \pi)^{2}} \int_{-\infty}^{\infty} \frac{d \omega}{2 \pi} \tanh \frac{\omega}{2 T} \operatorname{tr}\left(\gamma^{0} A(\omega, \vec{k})\right) .
$$

Substituting the spectral function eq. (69) and integrating over momenta, we obtain

$$
n=-\frac{2|q h| h_{1} v_{F}}{\pi} \int_{-\infty}^{\infty} \frac{d \omega}{2 \pi} \tanh \frac{\omega}{2 T} \Sigma_{2}(\omega) \sum_{l=0}^{\infty} \alpha_{l}\left(\frac{1}{\left(\tilde{\omega}-E_{l}\right)^{2}+\Sigma_{2}^{2}(\omega)}+\left(E_{l} \rightarrow-E_{l}\right)\right),
$$


where the degeneracy factor is $\alpha_{0}=1$ for the lowest Landau level and $\alpha_{l}=2$ for the higher Landau levels $l \geq 1$, $\tilde{\omega}=\omega+\varepsilon_{F}+\Sigma_{1}(\omega)$. Integrating over frequencies and taking into account that $\Sigma_{2}$ is effectively very small near the Fermi surface, we obtain

$$
n=\frac{|q h| h_{1} v_{F}}{\pi} \times 2\left(\tanh \frac{\varepsilon_{F}}{2 T}+\sum_{l=1}^{\infty}\left(\tanh \frac{\varepsilon_{F}+E_{l}}{2 T}+\tanh \frac{\varepsilon_{F}-E_{l}}{2 T}\right)\right) .
$$

Comparing with eq. (79), we obtain the relation $\left|\nu_{h}\right|=\frac{\pi}{|q h| h_{1} v_{F}} n$. When the Fermi energy vanishes $\left(\varepsilon_{F}=0\right)$, the spectral function eq. (69) is even in $\omega$. From eq. (88), the carrier density of stable quasiparticles vanishes when $\varepsilon_{F}=0$. At the end of this section, we discuss a situation with no stable charge carriers and physical consequences of it.
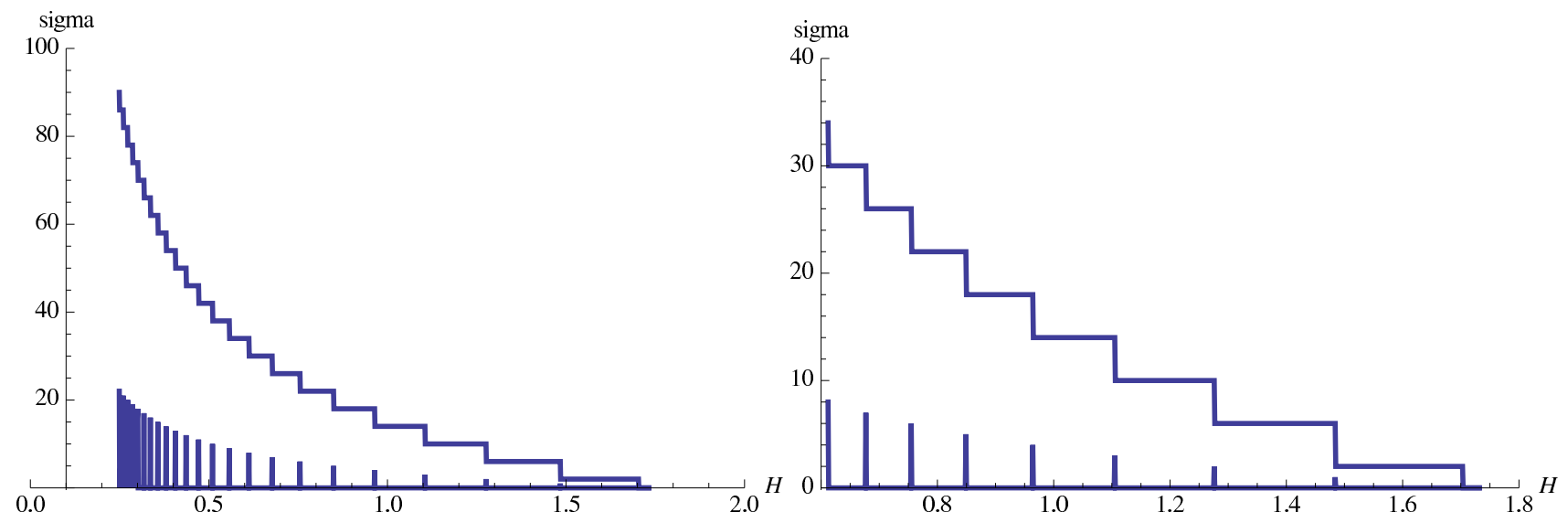

FIG. 12: Hall conductivity $\sigma_{x y}$ and longitudinal conductivity $\sigma_{x x}$ vs. the magnetic field $h \rightarrow H$ at $T=0$ (we set $g_{F}=1$, $\left.q=\frac{15}{\sqrt{3}}\right)$. Contribution from the first Fermi surface is taken. By decreasing the magnetic field, the Fermi surface crosses the Landau levels producing the Hall conductivity plateaus characteristic for IQHE. Longitudinal conductivity has picks at the beginning of each plateau. The right panel is a zoom-in for large $h$ of the left one.

Eqs. (779,89) are obtained assuming that the states are localized around the Landau levels. In QHE models, impurities are added to prevent the states from 'spilling' between the Landau levels and to provide the necessary occupation number of the levels. In our holographic calculations, however, the complex self-energy arises not from the impurities but from various scattering processes into the black hole. Here, the $\operatorname{limit} \operatorname{Im} \Sigma \rightarrow 0$ has been considered, which corresponds to a simplified field theory model [26] (the cited reference also considers the case with impurities). This approximation suffices to obtain the integer QHE [26] and for our initial studies of the fractional QHE. We leave the implementation of a physical model with impurities for future work.

\section{B. Fractional quantum Hall effect}

In a holographic setting, using the AdS geometry is equivalent to a calculation in a box. Therefore, for large enough fermion charge $q$, there are multiple Fermi surfaces as shown in Figs. 6 10. Labelling the Fermi surfaces with $\nu>\frac{1}{2}$ by $m=1,2, \ldots$, we represent the spectral function $A(\omega, \vec{k})$ as a sum over the spectral functions of individual Fermi surfaces given by eq. (69) [21]. Ignoring the mixing term, the DC conductivity becomes a direct sum over the individual conductivities. By decreasing the magnetic field, new Fermi surfaces gradually appear as can be seen in Figs. 677, Therefore the conductivity tensor is

$$
\sigma_{i j}=\sum_{m} \sigma_{i j}^{(m)} \theta\left(h_{\max }^{(m)}-h\right)
$$

where $\sigma_{i j}^{(m)}$ involves the Fermi momentum $k_{F}^{(m)}$ and velocity $v_{F}^{(m)}$, respectively; at the maximum magnetic field $h_{m a x}^{(m)}$ a new $k_{F}^{(m)}$ opens up; $h_{\max }^{(m)}$ is found numerically. 
Including one, two, three and four Fermi surfaces, we obtain the following quantization rule for the filling factor in the Hall conductivity:

$$
\begin{aligned}
& 1 \mathrm{FS}: \nu_{h}=2(1+2 n), \text { plateaus } \rightarrow 2,6,10, \ldots, \\
& 2 \mathrm{FS}^{\prime} \mathrm{s}: \nu_{h}=4(1+n+k), \text { plateaus } \rightarrow 4,8,12, \ldots, \\
& 3 \mathrm{FS}^{\prime} \mathrm{s}: \nu_{h}=2(3+2(n+k+p)), \text { plateaus } \rightarrow 6,10,14, \ldots, \\
& 4 \mathrm{FS}^{\prime} \mathrm{s}: \nu_{h}=4(2+n+k+p+r), \text { plateaus } \rightarrow 8,12,16, \ldots,
\end{aligned}
$$

with $n, k, p, r=0,1, \ldots$ An odd number of Fermi surfaces produces the plateaus present in the IQHE, while an even number of Fermi surfaces produces the additional plateaus appearing in the fractional QHE (FQHE). For a large enough fermion charge $q$, many Fermi surfaces contribute, and the primary effect of the change in $H$ is the opening of a new Fermi surface, rather than the occupation of the next plateau. Thus at large $q$ we expect a filling fraction pattern at large $h$ to become

$$
\nu_{h}= \pm 2 j
$$

where $j=1,2, \ldots$ is the effective Landau level index counting the number of contributing Landau levels. This is indeed observed in the FQHE at strong magnetic fields. The quantization rule (91) persists as long as new Fermi surfaces open up with decreasing $h$. However, the first two plateaus present in the FQHE $\nu_{h}=0, \pm 1$ are absent in eq. (92). In order to get the Hall plateau $\nu_{h}= \pm 1$, the mixing term between two Fermi surfaces should probably be taken into account (incoherent superposition), whereas the conductivity (90) includes the diagonal terms only. We discuss the issue with $\nu_{h}=0$ further.

In Fig. (13), we plot the Hall and longitudinal conductivities at $T=0$ with three Fermi surfaces contributing (eq. 90), where the individual conductivities $\sigma^{(m)}$ are given by eq. (79). We fit the Fermi momenta by

$$
k_{F}^{(m)}=k_{F \max }^{(m)} \sqrt{1-\frac{h^{2}}{3}}+\delta^{(m)},
$$

with $k_{F \max }^{(1)}=12.96, \delta^{(1)}=0 ., k_{F \max }^{(2)}=10.29, \delta^{(2)}=1.5, k_{F \max }^{(3)}=9.75, \delta^{(3)}=3$, and use eq. (93) together with the numerical solutions for $v_{F}^{(m)}$ in Fig. 13. In Fig. 13, at strong magnetic fields, the Hall conductivity plateau $\nu_{h}=4$ originates from two Fermi surfaces together with the plateaus $\nu_{h}=2$ and $\nu_{h}=6$ when one and three Fermi surfaces contribute, respectively. As we decrease the magnetic field further, three Fermi surfaces produce plateaus characteristic for IQHE eq. (82). The longitudinal conductivity shows a Dirac delta-like peak at the beginning of each plateau. Since a finite contribution to the conductivity arises as one of the three Fermi surfaces crosses the next Landau level, the pattern is less regular (i. e., the plateaus have changing length) than in the case when only one Fermi surface contributes. In Fig. 13, we compare the Hall conductivities with one and three Fermi surfaces participating. The irregular behavior of the Hall conductivity is explained naturally from the picture with multiple Fermi surfaces. Qualitatively similar regularity of the plateaus' length is seen in experiments on thin films of graphite at strong magnetic fields [24]. The actual physics behind, however, might be quite different as in this system multiple sheets of the Fermi surface arise due to the (hexagonal) lattice on the UV scale, an effect which is beyond the scope of our current model.

The somewhat regular pattern behind the irregular behavior can be understood as a consequence of the appearance of a new energy scale: the average distance between the Fermi levels. For the case of Fig. 13. we estimate it to be

$<\varepsilon_{F}^{(m)}-\varepsilon_{F}^{(m+1)}>=4.9$ with $m=1,2$. The authors of [26] explain the FQHE through the opening of a gap in the quasiparticle spectrum, which acts as an order parameter related to the particle-hole pairing and is enhanced by the magnetic field (magnetic catalysis). Here, the energy gap arises due to the participation of multiple Fermi surfaces.

A pattern for the Hall conductivity that is strikingly similar to Fig.(13) arises in the AA and AB-stacked bilayer graphene, which has different transport properties from the monolayer graphene [30], compare with Figs.(2,5) there. It is remarkable that the bilayer graphene also exhibits the insulating behavior in a certain parameter regime. This agrees with our findings of metal-insulating transition in our system.

\section{Metal-"strange metal" phase transition}

The previous discussion of conductivities and QHE is valid provided that the Fermi velocity is nonzero. However, we have shown that $v_{F}$ vanishes at relatively strong magnetic fields (for the first Fermi surface it happens at $h_{c}$ as in Fig. 8 and 11). In the AdS/CFT setting, the Fermi velocity vanishes when the IR anomalous dimension is $\nu=\frac{1}{2}$ signalling the onset of a nontrivial power law dispersion in Green's function $G^{-1}(\omega) \sim \omega-v_{f} k_{\perp}+\omega^{2 \nu}$ (the pole in the 


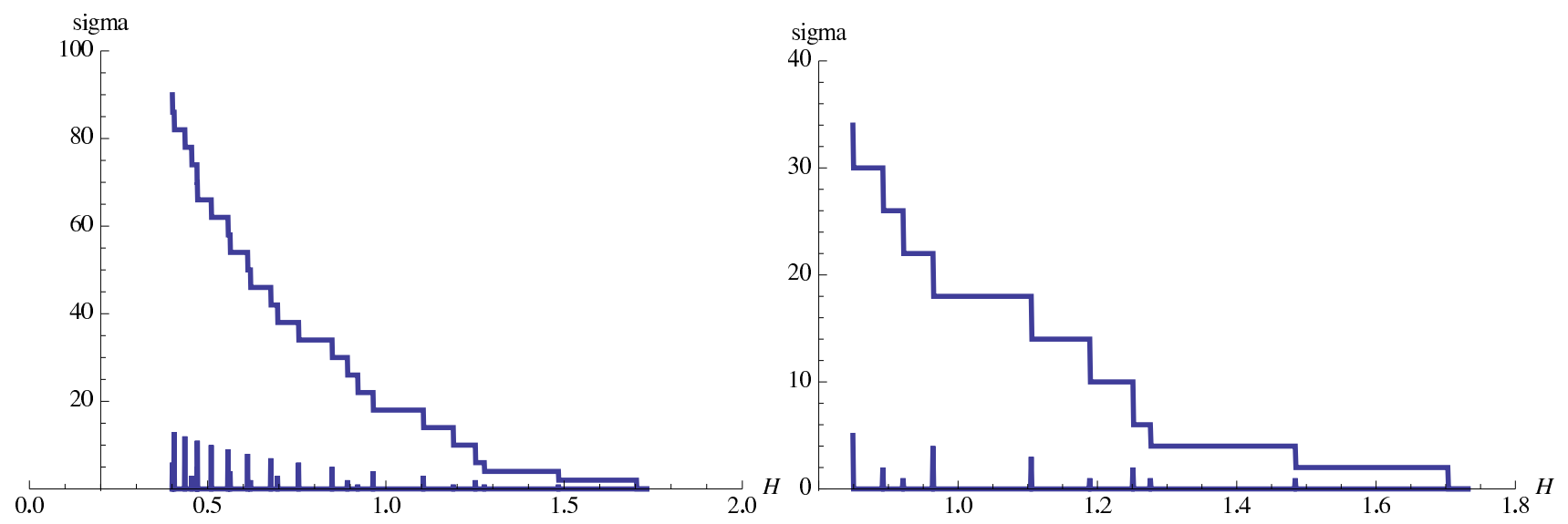

FIG. 13: Hall conductivity $\sigma_{x y}$ and longitudinal conductivity $\sigma_{x x}$ vs. the magnetic field $h \rightarrow H$ at $T=0$ (we set $g_{F}=1$, $\left.q=\frac{15}{\sqrt{3}}\right)$. Contribution from the first three Fermi surfaces are taken. At strong magnetic fields, the Hall conductivity plateau $\nu_{h}=4$ appears from two Fermi surfaces together with plateaus $\nu_{h}=2$ and $\nu_{h}=6$ when one and three Fermi surfaces contribute, respectively. This quantization rule is characteristic for the FQHE. At intermediate and weak magnetic fields, the Hall conductivity plateaus are produced as one of the three Fermi surfaces crosses the Landau levels, resulting in the quantization rule of the IQHE. Irregular pattern in the length of the plateaus is observed in the experiment on thin films of graphite at strong magnetic fields [24]. The right panel is a zoom-in for large $h$ of the left one.

self-energy $\Sigma \rightarrow G_{R}^{I R} \sim \omega^{2 \nu}$ and the pole in the prefactor of the linear term $\left.\sim \omega[7]\right)$. Vanishing of $v_{F}$ was observed in [21] at large enough fermion charge. Note that if $v_{F}$ is zero for some interval of the magnetic field, it leads to the Hall plateau with the filling factor $\nu_{h}=0$ present in FQHE.

The vanishing of the Fermi velocity of the stable quasiparticle leads to zero carrier density at leading order:

$$
v_{F}=0 \rightarrow n=0 .
$$

This means that all contribution to conductivity comes from the other terms, containing the contribution from the non-Fermi liquid excitations and the conformal regime. This qualitatively changes the transport properties of the system, as can be seen in Fig. 14.

The finite offset magnetic field has been observed in experiments on highly oriented pyrolitic graphite in magnetic fields [29]. In particular, analyzing the basal-plane resistivity an approximate scaling relation between the critical temperature of the metal-semiconducting transition and the magnetic field has been found $T_{c} \sim \sqrt{h-h_{c}}$. It suggests that at $T=0$, there is a threshold magnetic field $h_{c}$ above which the resistivity qualitatively changes. Interestingly, the existence of such a threshold magnetic field follows from the AdS/CFT calculations $\left(h_{c}\right.$ when $\left.\nu=\frac{1}{2}\right)$.

A phase transition is usually governed by an order parameter which exhibits a critical behavior. In our case, there is no such order parameter. However, it is interesting to note that the Fermi momentum, according to eq. (93), behaves as $k_{F} \sim \sqrt{h_{\max }-h}$, which is in line with the postulated critical behavior in the system, while the Fermi surface itself behaves as order parameter.

To obtain a complete picture of the metal-"strange metal" phase transition, one needs to perform calculations in the non-Fermi liquid regime taking into account the unstable quasiparticle pole. It is also necessary to study the temperature dependence of the DC conductivities $\sigma_{x y}(T)$ and $\sigma_{x x}(T)$. We leave it for the future study.

\section{ABSENCE OF THE SIGN PROBLEM IN HOLOGRAPHY.}

In this section we show that the fermion determinant in the gravity dual theory does not have a sign problem and hence can be simulated by a lattice Monte-Carlo algorithm. Up to recently, most of the work on AdS/CFT and applied holography focused on the classical gravity (leading $1 / N$ in field theory) limit. However, many thermodynamic and electric properties depend on matter fields (e. g., the electrical conductivity depends on whether or not the theory has a Fermi surface). In classical gravity, the Einstein-Maxwell sector decouples, and matter fields run in loops representing quantum oscillations. In order to include matter fields in the bulk, one needs to calculate loop corrections, which corresponds to going beyond the leading order in $1 / N$. A study of one-loop bulk physics was done in [31] and recently in [22]. It shows that analytical calculations of quantum corrections in the bulk are quite involved. The study of quantum oscillations in the gravity dual will likely improve our understanding of finite density systems in general. 


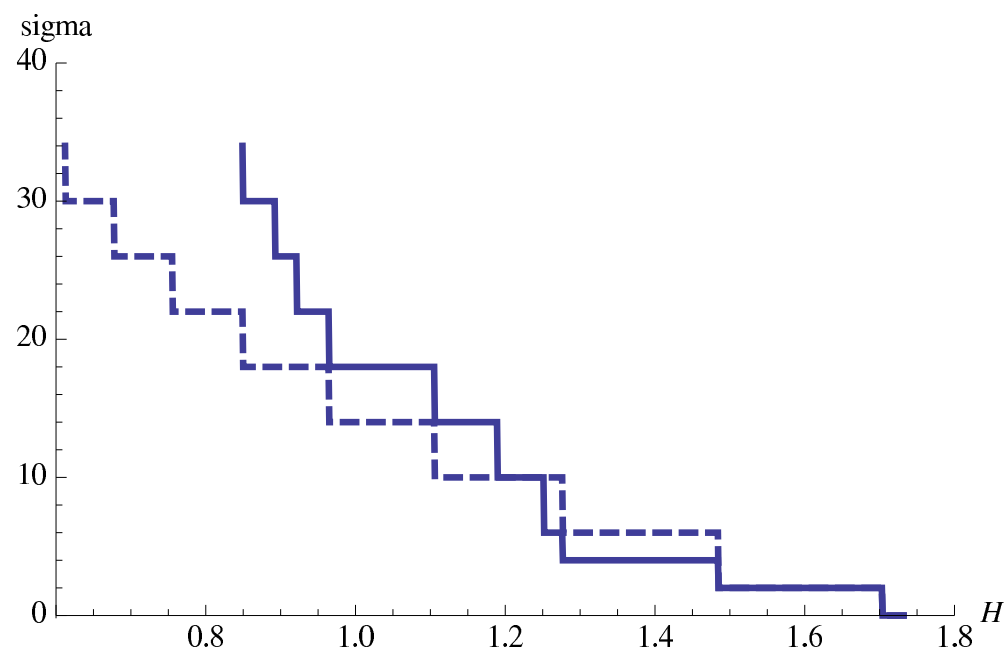

FIG. 14: Comparison of the Hall conductivities $\sigma_{x y}$ vs. the magnetic field $h \rightarrow H$ from one Fermi surface (dashed line) and from three Fermi surfaces (solid line). We set $g_{F}=1$ and $q=\frac{15}{\sqrt{3}}$. At strong magnetic fields, a new plateau $\nu_{h}=4$

appears in the multiple Fermi surface picture, yielding a pattern characteristic of FQHE.

As is well known, a finite density field theory in most cases cannot be simulated on the lattice because of the infamous sign problem [1]. In the field theory action, chemical potential is introduced via the term $\bar{\psi} \mu \gamma^{0} \psi$ which is Hermitian and therefore gives a complex determinant. At the same time, in the bulk action, finite density is introduced through the electrically charge black hole, and does not involve even matter fields. This is the reason why the applied holography gives universal predictions. In the leading order, the minimal gravitational dual at finite density and temperature is the electrically charged AdS-Reissner-Nordström black hole, where only the metric and Maxwell fields are present. Therefore, the Einstein-Maxwell sector gives results which do not depend, for example, on the charge and mass of matter fields in the gravitational bulk spacetime, i. e. are universal for a class of field theories with different charge and scaling dimensions of the operators. The fact that the chemical potential enters via the electric field in the covariant derivative leads to the real and positive definite fermion determinant which is suitable for lattice simulations. We show it formally below.

In a semiclassical approach to gravity, the action includes the Einstein-Maxwell sector $S_{g}$ with fields collectively denoted as $g, A$ in eq. (1) and the matter sector with the fermion fields $S_{\psi}$, eq. (21). The latter is given as (Euclidean signature):

$$
S_{\psi}=\int d^{4} x_{E} \sqrt{-g_{E}} \bar{\psi}(D+m) \psi
$$

where $D \equiv \Gamma_{E}^{M} \mathcal{D}_{M}$ and the covariant derivative is $\mathcal{D}_{M}=\partial_{M}+\frac{1}{4} \omega_{a b M} \Gamma^{a b}-i q A_{M}$. We can always scale away the spin connection by redefining the spinor field as in eq. A6). Finite density is described by the electrically charged black hole with charge $Q$, that generates the imaginary time component of the vector potential $A_{t_{E}}$ (eq. 17). Radial profile of the vector potential $A_{t_{E}}=\mu\left(1-\frac{1}{r}\right)$ (in dimensionless units) ensures a finite chemical potential at the field theory boundary $A_{t_{E}} \rightarrow \mu$ at $r \rightarrow \infty$, where $\mu=g_{F} Q$ (in dimensionless units). Integrating out the fermion fields, the gravitational partition function can be written schematically as

$$
Z=\sum_{g_{*}, A_{*}} \operatorname{det}\left(D\left(g_{*}, A_{*}\right)+m\right) \mathrm{e}^{-S_{g}\left[g_{*}, A_{*}\right]}
$$

where $S_{g}$ is the Euclidean gravitational action at the saddle points $g_{*}, A_{*}$. The determinant describes fluctuations about the saddle point solution $g_{*}, A_{*}$ and corresponds to $1 / N$ correction to the large $N$ limit of a dual gauge theory. Because the Euclidean gamma matrices are Hermitian by convention (the signature of the metric fixes the hermiticity), we have $\Gamma_{E}^{0 \dagger}=\Gamma_{E}^{0}$ and $\Gamma_{E}^{i \dagger}=\Gamma_{E}^{i}$ with $i=1,2,3$, so the covariant derivative is anti-Hermitian. Now it remains to show that the determinant of this anti-Hermitian differential operator is real and positive definite 32].

Using the anti-commutation relations $\left\{\Gamma_{E}^{5}, \Gamma_{E}^{0, i}\right\}=0$, we have

$$
\Gamma_{E}^{5} D \Gamma_{E}^{5}=-D=D^{\dagger},
$$


where $D \equiv D(g, A)$. Therefore the determinant

$$
\operatorname{det} D=\operatorname{det}\left(\Gamma_{E}^{5} D \Gamma_{E}^{5}\right)=\operatorname{det} D^{\dagger}=(\operatorname{det} D)^{*},
$$

is real. To show the positive definiteness, we remind the reader that the eigenmodes of an anti-Hermitian derivative operator come in pairs. If $(\lambda, \psi)$ is an eigenmode of $D$,

$$
D \psi=\lambda \psi,
$$

then, from eq. (99):

$$
D\left(\Gamma_{E}^{5} \psi\right)=(-\lambda)\left(\Gamma_{E}^{5} \psi\right),
$$

so $\left(-\lambda, \Gamma_{E}^{5} \psi\right)$ is also an eigenmode of $D$. Due to anti-hermiticity, from eq. (97):

$$
D\left(\Gamma_{E}^{5} \psi\right)=\lambda^{*}\left(\Gamma_{E}^{5} \psi\right),
$$

this eigenvalue is pure imaginary (or zero), $-\lambda=\lambda^{*}$. The determinant is a product of all the paired eigenvalues,

$$
\operatorname{det}(D+m) \rightarrow \Pi_{i}\left(\lambda_{i}+m\right)\left(-\lambda_{i}+m\right)=\Pi_{i}\left(\left|\lambda_{i}+m\right|^{2}\right),
$$

which is positive definite (or zero).

In field theory, the eigenmodes of the operator $D+\mu \gamma_{E}^{4}+m$ still come in pairs $(\lambda, \psi)$ and $\left(-\lambda, \gamma_{E}^{5} \psi\right)$. However, since $\mu \gamma_{E}^{4}$ is Hermitian, $\lambda$ is no longer pure imaginary, and therefore $\operatorname{det}\left(D+\mu \gamma_{E}^{4}+m\right)$ is not necessarily positive. The sign problem occurs when det is negative for some gauge configurations, or, in other words, it is generically present when considering interacting matter at finite density.

\section{CONCLUSIONS}

We have studied strongly coupled electron systems in the magnetic field focussing on the Fermi level structure, using the AdS/CFT correspondence. These systems are dual to Dirac fermions placed in the background of the electrically and magnetically charged AdS-Reissner-Nordström black hole. At strong magnetic fields the dual system "lives" near the black hole horizon, which substantially modifies the Fermi level structure. As we dial the magnetic field higher, the system exhibits the non-Fermi liquid behavior and then crosses back to the conformal regime. In our analysis we have concentrated on the the Fermi liquid regime and obtained the dependence of the Fermi momentum $k_{F}$ and Fermi velocity $v_{F}$ on the magnetic field. Remarkably, $k_{F}$ exhibits the square root behavior, with $v_{F}$ staying close to the speed of light in a wide range of magnetic fields, while it rapidly vanishes at a critical magnetic field which is relatively high. Such behavior indicates that the system may have a phase transition.

The magnetic system can be rescaled to a zero-field configuration which is thermodynamically equivalent to the original one. This simple result can actually be seen already at the level of field theory: the additional scale brought about by the magnetic field does not show up in thermodynamic quantities meaning, in particular, that the behavior in the vicinity of quantum critical points is expected to remain largely uninfluenced by the magnetic field, retaining its conformal invariance. In the light of current condensed matter knowledge, this is surprising and might in fact be a good opportunity to test the applicability of the probe limit in the real world: if this behavior is not seen, this suggests that one has to include the backreaction to metric to arrive at a realistic description.

In the field theory frame, we have calculated the DC conductivity using $k_{F}$ and $v_{F}$ values extracted from holography. The holographic calculation of conductivity that takes into account the fermions corresponds to the corrections of subleading order in $1 / N$ in the field theory and is very involved [15]. As we are not interested in the vertex renormalization due to gravity (it does not change the magnetic field dependence of the conductivity), we have performed our calculations directly in the field theory with AdS gravity-dressed fermion propagators. Instead of controlling the occupancy of the Landau levels by changing the chemical potential (as is usual in non-holographic setups), we have controlled the filling of the Landau levels by varying the Fermi energy level through the magnetic field. At zero temperature, we have reproduced the integer QHE of the Hall conductivity, which is observed in graphene at moderate magnetic fields. While the findings on equilibrium physics (Landau quantization, magnetic phase transitions and crossovers) are within expectations and indeed corroborate the meaningfulness of the AdS/CFT approach as compared to the well-known facts, the detection of the QHE is somewhat surprising as the spatial boundary effects are ignored in our setup. We plan to address this question in further work.

Interestingly, the AdS geometry produces several Fermi surfaces. Theories where the gravity duals have larger fermion charge $q$ posses more Fermi surfaces. We find that, in a multi-Fermi surface picture, the Hall conductivity is 
quantized in a way reminiscent of fractional QHE. By reducing the magnetic field, new Fermi surfaces open up and the quantization of Hall conductivity alternates between two different patterns, corresponding to odd and even number of Fermi surfaces. It turns out that odd number of the Fermi surfaces results in IQHE plateaus, while even number of surfaces gives new plateaus characteristic for the FQHE. In a multi-Fermi surface picture, the quantum Hall plateaus show a less regular pattern that agrees with experiments on thin graphite in strong magnetic field [24]. In our model it happens due to the fact that as one of several Fermi surfaces crosses the Landau level, the Hall conductivity jumps to a new plateau. This process is not synchronized between different Fermi surfaces. We associate the average distance between the Fermi levels with the energy gap usually arising in the FQHE.

Notably, the AdS-Reissner-Nordström black hole background gives a vanishing Fermi velocity at high magnetic fields. It happens at the point when the IR conformal dimension of the corresponding field theory is $\nu=\frac{1}{2}$, which is the borderline between the Fermi and non-Fermi liquids. Vanishing Fermi velocity was also observed at high enough fermion charge [21]. As in [21], it is explained by the red shift on the gravity side, because at strong magnetic fields the fermion wavefunction is supported near the black hole horizon modifying substantially the Fermi velocity. In our model, vanishing Fermi velocity leads to zero occupancy of the Landau levels by stable quasiparticles that results in vanishing regular Fermi liquid contribution to the Hall conductivity and the longitudinal conductivity. The dominant contribution to both now comes from the non-Fermi liquid and conformal contributions. We associate such change in the behavior of conductivities with a metal-"strange metal" phase transition. Experiments on highly oriented pyrolitic graphite support the existence of a finite "offset" magnetic field $h_{c}$ at $T=0$ where the resistivity qualitatively changes its behavior [29]. At $T \neq 0$, it has been associated with the metal-semiconducting phase transition [29]. It is worthwhile to study the temperature dependence of the conductivity in order to understand this phase transition better.

Finally, we suggest as a possibly interesting extension of the current AdS/CFT methodology to compute the gravity dual of the finite density matter in Monte-Carlo lattice simulations. This is possible since the sign problem does not arise in the holographic setting of a finite density system. Unlike the conventional field theory setup, finite density in holography is introduced through an electrically charged black hole, and does not involve matter fields (this is also the reason why holography gives universal results: it does not depend on the expectation values of matter fields at the leading order). In the gravity geometry, Dirac fermions are coupled minimally to the electric field via the covariant derivative. We have shown that the covariant derivative is anti-Hermitian in Euclidean signature, leading to the real and positive definite fermion determinant. This makes it possible to simulate finite density systems on the lattice in the AdS-gravity geometry using the curved space-time lattice formulation [33]. The simplest holographic setup which describes a finite charge density system includes a local $U(1)$ gauge symmetry. Finite density systems with global $U(1)$ symmetry can not be simulated numerically in field theory due to the problem with the Gauss law in the lattice formulation. Another important advantage of performing Monte-Carlo simulation is that it includes the quantum fluctuations for the gauge and gravitational field. So far most calculations have been done in the probe limit, with the frozen background for the metric and gauge fields. Analytic calculations which include backreaction are usually involved and are done in the next to leading order, e.g. [8]. Holographic lattice calculations allow to consider dynamical gauge and gravity fields with matter, which mimics complicated strong interactions in finite density systems and opens a way toward studying novel state of matter and instability mechanisms.

\section{Acknowledgements}

We thank Tom Faulkner, Michael Fromm, Tom Hartman, Sean Hartnoll, Nabil Iqbal, Hong Liu, Mark Mezei, Hiranmaya Mishra, Volodya Miransky, Yusuke Nishida, Owe Philipsen, Igor Shovkovy, Tian Zhang, Darius Sadri, Chang Yu Hou, Andrej Mesaroš and Vladimir Juričić for helpful inputs and discussions. The work was supported in part by the Alliance program of the Helmholtz Association, contract HA216/EMMI "Extremes of Density and Temperature: Cosmic Matter in the Laboratory" and by ITP of Goethe University, Frankfurt (E. Gubankova), by a VIDI Innovative Research Incentive Grant (K. Schalm) from the Netherlands Organization for Scientific Research (NWO), by a Spinoza Award (J. Zaanen) from the Netherlands Organization for Scientific Research (NWO) and the Dutch Foundation for Fundamental Research of Matter (FOM). K. Schalm thanks the Galileo Galilei Institute for Theoretical Physics for the hospitality and the INFN for partial support during the completion of this work. 


\section{Appendix A: Dirac equation in magnetic field}

Here we solve analytically the part of the Dirac equation which depends on magnetic field and space-time coordinates of the boundary theory. The free spinor action in the geometry given by eq. (2) and in the presence of magnetic field (3) is given by eq. (21).

Using the translational invariance,

$$
\psi(t, x, y, r)=\int d \omega d k \mathrm{e}^{-i \omega t+i k y} \psi(\omega, k, x, r),
$$

with $k \equiv k_{y}$, the Dirac equation (eq. 23) can be written as

$$
\begin{gathered}
\left(\frac{1}{\sqrt{-g_{t t}}} \Gamma^{\hat{t}}\left(-i \omega+\frac{1}{2} \omega_{\hat{t} \hat{r} t} \Gamma^{\hat{t} \hat{r}}-i q A_{t}(r)\right)+\frac{1}{\sqrt{g_{r r}}} \Gamma^{\hat{r}} \partial_{r}+\frac{1}{\sqrt{g_{i i}}} \Gamma^{\hat{x}}\left(\partial_{x}+\frac{1}{2} \omega_{\hat{x} \hat{r} x} \Gamma^{\hat{x} \hat{r}}\right)\right. \\
\left.+\frac{1}{\sqrt{g_{i i}}} \Gamma^{\hat{y}}\left(i k+\frac{1}{2} \omega_{\hat{y} \hat{r} y} \Gamma^{\hat{y} \hat{r}}-i q A_{y}(x)\right)-m\right) \psi(\omega, k, x, r)=0
\end{gathered}
$$

where $g_{i i} \equiv g_{x x}=g_{y y}$, and $A_{t}(r)=\mu\left(1-r_{0} / r\right), A_{y}(x)=h x$. From the torsion-free condition, $\omega_{b}^{a} \wedge e^{b}=-d e^{a}$, we find the spin connection [34] for the metric 2

$$
\omega_{\hat{t} \hat{r}}=-\frac{\partial_{r}\left(\sqrt{-g_{t t}}\right)}{\sqrt{g_{r r}}} d t, \quad \omega_{\hat{i} \hat{r}}=\frac{\partial_{r}\left(\sqrt{g_{i i}}\right)}{\sqrt{g_{r r}}} d x^{i},
$$

where $i=x, y$. Note that

$$
-\Gamma^{\hat{t}} \Gamma^{\hat{t} \hat{r}}=\Gamma^{\hat{x}} \Gamma^{\hat{x} \hat{r}}=\Gamma^{\hat{y}} \Gamma^{\hat{y} \hat{r}}=\Gamma^{\hat{r}}
$$

and

$$
\begin{aligned}
\frac{1}{4} e_{\hat{a}}^{M} \Gamma^{\hat{a}} \omega_{\hat{b} \hat{c} M} \Gamma^{\hat{b} \hat{c}} & =\frac{1}{4} \frac{1}{\sqrt{-g_{t t}}} \frac{\partial_{r}\left(\sqrt{-g_{t t}}\right)}{\sqrt{g_{r r}}} \Gamma^{\hat{r}}+\frac{2}{4} \frac{1}{\sqrt{g_{i i}}} \frac{\partial_{r} \sqrt{g_{i i}}}{\sqrt{g_{r r}}} \Gamma^{\hat{r}} \\
& =\frac{1}{\sqrt{g_{r r}}} \Gamma^{\hat{r}} \partial_{r} \ln \left(-\frac{g}{g_{r r}}\right)^{1 / 4},
\end{aligned}
$$

where $g$ is the determinant of the metric. Therefore, we can rescale the spinor field:

$$
\psi=\left(-\frac{g}{g_{r r}}\right)^{-1 / 4} \Phi
$$

and remove the spin connection completely. The new covariant derivative does not contain the spin connection so $\mathcal{D}_{M}^{\prime}=\partial_{M}-i q A_{M}$.

In new field variables, the Dirac equation is given by

$$
\begin{gathered}
\left(\frac{\sqrt{g_{i i}}}{\sqrt{g_{r r}}} \Gamma^{\hat{r}} \partial_{r}-\frac{\sqrt{g_{i i}}}{\sqrt{-g_{t t}}} \Gamma^{\hat{t}} i\left(\omega+\mu_{q}\left(1-\frac{r_{0}}{r}\right)\right)-\sqrt{g_{i i}} m+\Gamma^{\hat{x}} \partial_{x}\right. \\
\left.+\Gamma^{\hat{y}} i(k-q h x)\right) \Phi(\omega, k, x, r)=0
\end{gathered}
$$

with $\mu_{q} \equiv \mu q$. We separate the $x$ - and $r$-dependent parts:

$$
\begin{aligned}
& P(r)=\frac{\sqrt{g_{i i}}}{\sqrt{g_{r r}}} \Gamma^{\hat{r}} \partial_{r}-\frac{\sqrt{g_{i i}}}{\sqrt{-g_{t t}}} \Gamma^{\hat{t}} i\left(\omega+\mu_{q}\left(1-\frac{r_{0}}{r}\right)\right)-\sqrt{g_{i i}} m, \\
& Q(x)=\Gamma^{\hat{x}} \partial_{x}+\Gamma^{\hat{y}} i(k-q h x),
\end{aligned}
$$

and the Dirac equation is

$$
(P(r)+Q(x)) \Phi=0 .
$$


Even though $[P(r), Q(x)] \neq 0$, one can find a transformation matrix $U$ such that $[U P(r), U Q(x)]=0$, and then look for common eigenvectors of $U P(r)$ and $U Q(x)$ as they are commuting Hermitian operators, i. e., the Dirac equation reads

$$
U P(r) \Phi_{l}=-U Q(x) \Phi_{l}=\lambda_{l} \Phi_{l},
$$

where $l$ labels the Landau levels. We use $l$ for the Landau index, so as not to confuse it with the Matsubara frequency index $n$. Transformation matrix $U$ should satisfy the conditions

$$
\left\{U, \Gamma^{\hat{r}}\right\}=0, \quad\left\{U, \Gamma^{\hat{t}}\right\}=0, \quad\left[U, \Gamma^{\hat{x}}\right]=0, \quad\left[U, \Gamma^{\hat{y}}\right]=0,
$$

which do not fix $U$ completely. It is convenient to use the following basis [7]:

$$
\begin{aligned}
& \Gamma^{\hat{r}}=\left(\begin{array}{cc}
-\sigma^{3} & 0 \\
0 & -\sigma^{3}
\end{array}\right), \quad \Gamma^{\hat{t}}=\left(\begin{array}{cc}
i \sigma^{1} & 0 \\
0 & i \sigma^{1}
\end{array}\right), \quad \Gamma^{\hat{x}}=\left(\begin{array}{cc}
-\sigma^{2} & 0 \\
0 & \sigma^{2}
\end{array}\right), \\
& \Gamma^{\hat{y}}=\left(\begin{array}{cc}
0 & \sigma^{2} \\
\sigma^{2} & 0
\end{array}\right), \quad \Gamma^{\hat{5}}=\left(\begin{array}{cc}
0 & i \sigma^{2} \\
-i \sigma^{2} & 0
\end{array}\right) .
\end{aligned}
$$

Note, that the following relation holds

$$
\Gamma^{\hat{5}}=\Gamma^{\hat{0}} \Gamma^{\hat{1}} \Gamma^{\hat{2}} \Gamma^{\hat{3}}
$$

as expected, with $0 \rightarrow t, 1 \rightarrow x, 2 \rightarrow y, 3 \rightarrow r$. In the representation of eq. (A12), we can choose

$$
U=\left(\begin{array}{cc}
-i \sigma^{2} & 0 \\
0 & -i \sigma^{2}
\end{array}\right)
$$

We split the 4-component spinors into two 2-component spinors (we do not write zero entries) $F=\left(F_{1}, F_{2}\right)^{T}$ where the index $\alpha=1,2$ is the Dirac index of the boundary theory, using projectors

$$
\Pi_{\alpha}=\frac{1}{2}\left(1-(-1)^{\alpha} \Gamma^{\hat{r}} \Gamma^{\hat{t}} \Gamma^{\hat{1}}\right), \quad \alpha=1,2, \quad \Pi_{1}+\Pi_{2}=1,
$$

which commute with the Dirac operator of eq. (37), and $F_{\alpha}=\Pi_{\alpha} \Phi, \alpha=1,2$, decouple from each other. Gamma matrices in eq. (A12) were chosen in such a way that this decoupling is possible.

Writing the Dirac equation (A10) for $F=\left(F_{1}, F_{2}\right)^{T}$, we have

$$
\begin{aligned}
& \left(-\frac{\sqrt{g_{i i}}}{\sqrt{g_{r r}}} \sigma^{1} \partial_{r}+\sqrt{g_{i i}} i \sigma^{2} m-\frac{\sqrt{g_{i i}}}{\sqrt{-g_{t t}}} \sigma^{3}\left(\omega+\mu_{q}\left(1-r_{0} / r\right)\right)-\lambda_{n}\right) \otimes 1\left(\begin{array}{c}
F_{1} \\
F_{2}
\end{array}\right)=0 \\
& 1 \otimes\left(\begin{array}{cc}
i \partial_{x}+\lambda_{l} & (k-q h x) \\
(k-q h x) & -i \partial_{x}+\lambda_{l}
\end{array}\right)\left(\begin{array}{c}
F_{1} \\
F_{2}
\end{array}\right)=0,
\end{aligned}
$$

where in $X \otimes Y, X$ acts inside $F_{1}$ or $F_{2}$ and $Y$ acts between $F_{1}$ and $F_{2}$. In eq. (A16), the 1 in the first equation shows that there is no mixing of $F_{1}$ and $F_{2}$ by the operator $U P(r)$ and the 1 in the second equation shows that there is no mixing inside $F_{1}$ or $F_{2}$ by the operator $U Q(x)$. Therefore, the solution can be represented as

$$
\left(\begin{array}{c}
F_{1} \\
F_{2}
\end{array}\right)=\left(\begin{array}{l}
f_{l}^{(1)}(r) g_{l}^{(1)}(x) \\
f_{l}^{(2)}(r) g_{l}^{(1)}(x) \\
f_{l}^{(1)}(r) g_{l}^{(2)}(x) \\
f_{l}^{(2)}(r) g_{l}^{(2)}(x)
\end{array}\right) .
$$

We do not write explicitly the dependence on $\omega$ and $k$. It is convenient to make a unitary transformation:

$$
\left(\begin{array}{c}
\zeta^{(1)} \\
\zeta^{(2)}
\end{array}\right)=M\left(\begin{array}{c}
g^{(1)} \\
g^{(2)}
\end{array}\right), M=\left(\begin{array}{cc}
1 & -i \\
-i & 1
\end{array}\right)
$$

Dirac equations for each component are written as:

$$
\begin{aligned}
\left(\frac{\sqrt{g_{i i}}}{\sqrt{g_{r r}}} \partial_{r}+\sqrt{g_{i i}} m\right) f_{l}^{(1)}(r)+\left(-\frac{\sqrt{g_{i i}}}{\sqrt{-g_{t t}}}\left(\omega+\mu_{q}\left(1-r_{0} / r\right)\right)+\lambda_{l}\right) f_{l}^{(2)}(r) & =0 \\
\left(\frac{\sqrt{g_{i i}}}{\sqrt{g_{r r}}} \partial_{r}-\sqrt{g_{i i}} m\right) f_{l}^{(2)}(r)+\left(\frac{\sqrt{g_{i i}}}{\sqrt{-g_{t t}}}\left(\omega+\mu_{q}\left(1-r_{0} / r\right)\right)+\lambda_{l}\right) f_{l}^{(1)}(r) & =0 \\
\left(\partial_{\tilde{x}}-\tilde{x}\right) \zeta^{(1)}+\tilde{\lambda}_{l} \zeta^{(2)} & =0 \\
\left(\partial_{\tilde{x}}+\tilde{x}\right) \zeta^{(2)}-\tilde{\lambda}_{l} \zeta^{(1)} & =0 .
\end{aligned}
$$


In equations A20 for the $x$-dependent part, we have rescaled $\tilde{x}=\sqrt{|q h|}\left(x-\frac{k}{q h}\right)$ with $k \equiv k_{y}$ and $\lambda_{l}=\sqrt{|q h|} \tilde{\lambda}_{l}$. The second order ordinary differential equations

$$
-\partial_{\tilde{x}}^{2} \zeta^{(\rho)}+\tilde{x}^{2} \zeta^{(\rho)}-\tilde{\lambda}_{l}^{2} \zeta^{\rho}-(-1)^{\rho} \zeta^{(\rho)}=0,
$$

with $\rho=1,2$, are solved by substitution $\zeta^{(\rho)}=\mathrm{e}^{-\tilde{x}^{2} / 2} \tilde{\zeta}^{(\rho)}$. This is exactly the Schrödinger equation for a harmonic oscillator, so the eigenfunctions are Hermite polynomials and we obtain the following solutions, indexed by an integer $l \in \mathbb{Z}$ that is related to the eigenvalue $\lambda_{l}$ by $\lambda_{l}=\sqrt{2|q h| l}$ :

$$
\begin{aligned}
\zeta_{l}^{(1)}(\tilde{x}) & =N_{l-1} e^{-\tilde{x}^{2} / 2} H_{l-1}(\tilde{x}) \\
\zeta_{l}^{(2)}(\tilde{x}) & =N_{l} e^{-\tilde{x}^{2} / 2} H_{l}(\tilde{x}) .
\end{aligned}
$$

The normalization constant $N_{l}$ is proportional to $1 / \sqrt{2^{l} l !}$. Substituting the solutions from eq. A22 into the first order eigenvalue equation with $x$-dependence gives the following solutions:

$$
F_{l}=\left(\begin{array}{c}
f_{l}^{(1)}(r) \zeta_{l}^{(1)}(\tilde{x}) \\
f_{l}^{(2)}(r) \zeta_{l}^{(1)}(\tilde{x}) \\
f_{l}^{(1)}(r) \zeta_{l}^{(2)}(\tilde{x}) \\
-f_{l}^{(2)}(r) \zeta_{l}^{(2)}(\tilde{x})
\end{array}\right), \quad \lambda_{l}=\sqrt{2|q h| l}
$$

and

$$
\tilde{F}_{l}=\left(\begin{array}{c}
\tilde{f}_{l}^{(1)}(r) \zeta_{l}^{(1)}(\tilde{x}) \\
\tilde{f}_{l}^{(2)}(r) \zeta_{l}^{(1)}(\tilde{x}) \\
-\tilde{f}_{l}^{(1)}(r) \zeta_{l}^{(2)}(\tilde{x}) \\
\tilde{f}_{l}^{(2)}(r) \zeta_{l}^{(2)}(\tilde{x})
\end{array}\right), \quad \lambda_{l}=-\sqrt{2|q h| l} .
$$

Solving the first order $x$-dependent equation, we get the same eigenvalue, but slightly different eigenfunctions for different signs of $q h$. In particular, e. g., for $F$, the pairs $f^{(1)}\left(\zeta^{(1)}, \zeta^{(2)}\right)^{T}$ and $f^{(2)}\left(\zeta^{(1)},-\zeta^{(2)}\right)^{T}$ correspond to $q h>0$ and $q h<0$, respectively. Different sign of $q h$ stands for the positive and negative Landau level index $l$.

Finally, the general solution to the Dirac equation is given by a linear combination of eqs. (A23) and (A24):

$$
F_{\text {sol }}=\sum_{l}\left(a_{l} F_{l}+b_{l} \tilde{F}_{l}\right)
$$

Using the eigenvalues determined by eqs. A23 A24 in the equation for the radial part A19), we get

$$
\begin{gathered}
\left(-\frac{1}{\sqrt{g_{r r}}} \sigma^{3} \partial_{r}-m+\frac{1}{\sqrt{-g_{t t}}} \sigma^{1}\left(\omega+\mu_{q}\left(1-r_{0} / r\right)\right)\right. \\
\left.-\frac{1}{\sqrt{g_{i i}}} i \sigma^{2} \sqrt{2|q h| l}\right) \otimes 1\left(\begin{array}{c}
F_{1} \\
F_{2}
\end{array}\right)=0,
\end{gathered}
$$

with $l=0,1$, dots; and the same for $\tilde{F}$ replacing $\sqrt{2|q h| l} \rightarrow-\sqrt{2|q h| l}$. It coincides with eq. (A14) in [7] (Dirac equation at zero magnetic field) with the momentum replaced by the Landau level eigenvalue [22]

$$
k \rightarrow \pm \sqrt{2|q h| l} .
$$

Equation (A27) also gives a prescription on how to treat the limit of zero magnetic field $h \rightarrow 0$. The limit is to be taken keeping, e. g., $2|q h|(l+1) \equiv k_{F}^{2}$ fixed as $h \rightarrow 0$. In a compact form, the Dirac equation in a magnetic field (A7) is given by

$$
\begin{gathered}
\left(\frac{1}{\sqrt{g_{r r}}} \Gamma^{\hat{r}} \partial_{r}-\frac{1}{\sqrt{-g_{t t}}} \Gamma^{\hat{t}} i\left(\omega+q A_{t}\right)-m\right. \\
\left.-\frac{1}{\sqrt{g_{i i}}} U^{-1} \sqrt{2|q h| l}\right) F(r)=0,
\end{gathered}
$$

with $F=\left(F_{1}, F_{2}\right)^{T}, l=0,1, \ldots$, for $\tilde{F}$ replace $\sqrt{2|q h| l} \rightarrow-\sqrt{2|q h| l}$, and $U^{-1}$ is the matrix inverse to the matrix given by eq. (A14):

$$
U^{-1}=\left(\begin{array}{cc}
i \sigma^{2} & 0 \\
0 & i \sigma^{2}
\end{array}\right)
$$

which we use in the main text. 


\section{Appendix B: Spectral function}

In what follows we use the dimensionless variables (15] 17]). Following the analysis of [7], the flow of the Green's function is determined by

$$
G_{R}(\omega, l)=\left.\lim _{\epsilon \rightarrow 0} \epsilon^{-2 m}\left(\begin{array}{cc}
\xi_{+}^{(l)} & 0 \\
0 & \xi_{-}^{(l)}
\end{array}\right)\right|_{r=\frac{1}{\epsilon}}
$$

where $\xi_{+}^{(l)}(r)=\frac{f^{(2)}}{f^{(1)}}$ and $\xi_{-}^{(l)}(r)=\frac{\tilde{f}^{(2)}}{\tilde{f}^{(1)}}$ from the solutions A23 A24). In obtaining this relation, we absorbed the coefficients appearing in eq. A25 into the definitions of the radial functions. The functions $\xi_{ \pm}^{(l)}$ satisfy the following differential equation [7]:

$$
\sqrt{\frac{g_{i i}}{g_{r r}}} \partial_{r} \xi_{ \pm}^{(l)}=-2 m \sqrt{g_{i i}} \xi_{ \pm}^{(l)}+\left(u(r) \pm \lambda_{l}\right)^{2}\left(\xi_{ \pm}^{(l)}\right)^{2}+\left(u(r) \mp \lambda_{l}\right)
$$

with $u(r)$ given by

$$
u(r)=\sqrt{\frac{g_{i i}}{-g_{t t}}}\left(\omega+q A_{t}(r)\right) .
$$

Writing explicitly in the metric eq.(18), we have

$$
r^{2} \sqrt{f} \partial_{r} \xi_{ \pm}^{(l)}=-2 m r \xi_{ \pm}^{(l)}+\left(u(r) \pm \lambda_{l}\right)\left(\xi_{ \pm}^{(l)}\right)^{2}+\left(u(r) \mp \lambda_{l}\right)
$$

where $u(r)$ is given by

$$
u(r)=\frac{1}{\sqrt{f}}\left(\omega+\mu_{q}\left(1-\frac{1}{r}\right)\right)
$$

with $f=\frac{(r-1)^{2}\left(r^{2}+2 r+3\right)}{r^{4}}$ at $T=0$. Near the horizon $(r=1)$ the flow equation reduces to

$$
r^{2} \partial_{r} \xi_{ \pm}^{(l)}=\frac{1}{f}\left(\xi_{ \pm}^{(l)}+1\right)^{2}
$$

which due to the double zero in $f$ has a regular solution only if $\xi_{ \pm}(r=1)= \pm i$. Writing the radial equation in terms of $\xi$ and choosing the infalling boundary conditions fixes $\xi_{ \pm}^{(l)}(r=1)=i$.

The key quantity that we extract from the Green's function is the fermionic spectral function

$$
A\left(\omega, l_{x}, k_{y}\right)=\operatorname{Tr}\left(\operatorname{Im} G_{R}\left(\omega, l_{x}, k_{y}\right)\right)
$$

which we analyze in the main text of the paper.

[1] J. Zaanen, "Quantum Critical Electron Systems: The Uncharted Sign Worlds", Science 319, 1205 (2008); P. de Forcrand, "Simulating QCD at finite density", PoS LAT2009, 010 (2009), arXiv:1005.0539 [hep-lat]]; M. P. Lombardo, K. Splittorff, J. J. M. Verbaarschot, "Fluctuations, correlations and the sign problem in QCD ", PoS LAT2009, 171 (2009), arXiv:0912.3109 hep-lat]].

[2] S. A. Hartnoll, J. Polchinski, E. Silverstein and D. Tong, "Towards strange metallic holography", JHEP 1004, 120 (2010) arXiv:0912.1061 hep-th]]; S. A. Hartnoll, C. P. Herzog and G. T. Horowitz, "Holographic Superconductors", JHEP 0812, 015, (2008) arXiv:0810.1563 hep-th]].

[3] P. Kovtun, D. T. Son, A. O. Starinets, "Viscosity in Strongly Interacting Quantum Field Theories from Black Hole Physics ", Phys. Rev. Lett. 94, 111601 (2005), [arXiv:0405231[hep-th]].

[4] S. -S. Lee, "A non-Fermi liquid from a charged black hole: a critical Fermi ball", Phys. Rev. D 79, 086006 (2009) arXiv:0809.3402 hep-th]].

[5] M. Čubrović, J. Zaanen and K. Schalm, "String theory, quantum phase transitions and the emergent Fermi-liquid", Science 325, 439 (2009) arXiv:0904.1993 hep-th]];

[6] H. Liu, J. McGreevy and D. Vegh, "Non-Fermi liquids from holography", arXiv:0903.2477 [hep-th]. 
[7] T. Faulkner, H. Liu, J. McGreevy and D. Vegh, "Emergent quantum criticality, Fermi surfaces, and AdS2", arXiv:0907.2694 [hep-th]]; T. Faulkner, N. Iqbal, H. Liu, J. McGreevy and D. Vegh, "Holographic non-Fermi liquid fixed points", arXiv:1101.0597[hep-th]].

[8] S. A. Hartnoll and A. Tavanfar, "Electron stars for holographic metallic criticality", arXiv:1008.2828 hep-th]].

[9] S. A. Hartnoll, P. K. Kovtun, M. Mueller and S. Sachdev, "Theory of the Nernst effect near quantum phase transitions in condensed matter, and in dyonic black holes", Phys. Rev. B 76, 144502 (2007) arXiv:0706.3215 hep-th]].

[10] S. A. Hartnoll and P. Kovtun, "Hall conductivity from dyonic black holes", Phys. Rev. D 76, 066001 (2007), arXiv:0704.1160 hep-th]].

[11] P. Basu, J. Y. He, A. Mukherjee and H.-H. Shieh, "Holographic Non-Fermi Liquid in a Background Magnetic Field", [arXiv:10908.1436[hep-th]].

[12] T. Albash and C. V. Johnson, "Landau Levels, Magnetic Fields and Holographic Fermi Liquids", J. Phys. A: Math. Theor. 43, 345404 (2010) arXiv:1001.3700[hep-th]]; T. Albash and C. V. Johnson, "Holographic Aspects of Fermi Liquids in a Background Magnetic Field", J. Phys. A: Math. Theor. 43, 345405 (2010) arXiv:0907.5406 hep-th]].

[13] T. Albash and C. V. Johnson, "A holographic superconductor in an external magnetic field", JHEP 0809, 121 (2008) arXiv:0804.3466 hep-th]]. T. Albash and C. V. Johnson, "Phases of holographic superconductors in an external magnetic field", arXiv:0906.0519 hep-th]].

[14] N. Iqbal, H. Liu, M. Mezei and Q. Si "Quantum phase transitions in holographic models of magnetism and superconductors", arXiv:1003.0010 [hep-th]].

[15] T. Faulkner, N. Iqbal, H. Liu, J. McGreevy and D. Vegh, "From black holes to strange metals", arXiv:1003.1728[hep-th]].

[16] E. D'Hoker and P. Kraus, JHEP 1005, 083 (2010) arXiv:1003.1302 [hep-th]].

E. D'Hoker and P. Kraus, Class. Quant. Grav. 27, 215022 (2010) arXiv:1006.2573 [hep-th]].

[17] A. Auerbach, "Quantum Magnetism Approaches to Strongly Correlated Electrons ", arXiv:cond-mat/9801294.

[18] E. Gubankova, "Particle-hole instability in the $A d S_{4}$ holography", arXiv:1006.4789 hep-th]].

[19] J. L. Davis, P. Kraus and A. Shah, "Gravity Dual of a Quantum Hall Plateau Transition", JHEP 0811, 020 (2008) arXiv:0809.1876 hep-th]]; E. K. -Vakkuri, P. Kraus, "Quantum Hall Effect in AdS/CFT", JHEP 0809, 130 (2008) arXiv:0805.4643 hep-th]].

[20] A. H. MacDonald, "Introduction to the physics of the Quantum Hall regime", arXiv:cond-mat/9410047.

[21] T. Hartman and S. A. Hartnoll, "Cooper pairing near charged black holes", arXiv:1003.1918[hep-th]].

[22] F. Denef, S. A. Hartnoll and S. Sachdev, "Quantum oscillations and black hole ringing", arXiv:0908.1788 [hep-th]; F. Denef, S. A. Hartnoll and S. Sachdev, "Black hole determinants and quasinormal modes", arXiv:0908.2657 [hep-th].

[23] J. L. Noronha, I. A. Shovkovy, "Magnetization of Color-Flavor Locked Matter", arXiv:0710.2445[hep-ph]]; J. L. Noronha, I. A. Shovkovy, "Color-flavor locked superconductor in a magnetic field", Phys. Rev. D 76,105030 (2007) arXiv:0708.0307[hep-ph]].

[24] Y. Zhang, Z. Jiang, J. P. Small, M. S. Purewal, Y.-W. Tan, M. Fazlollahi, J. D. Chudow, J. A. Jaszczak, H. L. Stormer and P. Kim, "Landau Level Splitting in Graphene in High Magnetic Fields", Phys. Rev. Lett. 96, 136806 (2006) [arXiv:0602649[cond-mat]].

[25] M. A. V. Basagoiti, "Transport coefficients and ladder summation in hot gauge theories", Phys. Rev. D 66, 045005 (2002) arXiv:hep-ph/0204334; J. M. M. Resco, M. A. V. Basagoiti, "Color conductivity and ladder summation in hot QCD", Phys. Rev. D 63, 056008 (2001), arXiv:hep-ph/0009331.

[26] E. V. Gorbar, V. P. Gusynin, V. A. Miransky and I. A. Shovkovy, "Magnetic field driven metal-insulator phase transition in planar systems", Phys. Rev. B 66, 045108 (2002) [arXiv:0202422[cond-mat]]; V. P. Gusynin and S. G. Sharapov, "Transport of Dirac quasiparticles in graphene: Hall and optical conductivities", Phys. Rev. B 73, 245411 (2006) [arXiv:0512157[condmat]]; V. P. Gusynin, V. A. Miransky, S. G. Sharapov and I. A. Shovkovy, "Excitonic gap, phase transition, and quantum Hall effect in graphene", Phys. Rev. B 74, 195429 (2006) [arXiv:0605348[cond-mat]]; V. P. Gusynin and S. G. Sharapov, "Unconventional Integer Quantum Hall effect in graphene", Phys. Rev. Lett. 95, 146801 (2005) [arXiv:0506575[cond-mat]].

[27] E. V. Gorbar, V. A. Miransky, I. A. Shovkovy, "Dynamics in the normal ground state of dense relativistic matter in a magnetic field", arXiv:1101.4954 hep-th]].

[28] N. Iqbal and H. Liu, "Real-time response in AdS/CFT with application to spinors", Fortsch. Phys. 57, 367 (2009), arXiv:0903.2596 hep-th]].

[29] Y. Kopelevich, V. V. Lemanov, S. Moehlecke and J. H. S. Torrez, "Landau Level Quantization and Possible Superconducting Instabilities in Highly Oriented Pyrolitic Graphite", Fiz.Tverd.Tela 41, 2135 (1999) [Phys. Solid State 41, 1959 (1999)]; H. Kempa, Y. Kopelevich, F. Mrowka, A. Setzer, J. H. S. Torrez, R. Hoehne and P. Esquinazi, Solid State Commun. 115, 539 (2000); M. S. Sercheli, Y. Kopelevich, R. R. da Silva, J. H. S. Torrez and C. Rettori, Solid State Commun. 121, 579 (2002); Y. Kopelevich, P. Esquinazi, J. H. S. Torres, R. R. da Silva, H. Kempa, F. Mrowka, R. Ocana, "Metal-Insulator-Metal Transitions, Superconductivity and Magnetism in Graphite", Studies of H-Temp. Supercond. 45, 59 (2003), [arXiv:0209442 [cond-mat]].

[30] Y.-F. Hsu, G.-Y. Guo, "Anomalous integer quantum Hall effect in AA-stacked bilayer graphene", Phys. Rev. B 82, 165404 (2010), arXiv:1008.0748[cond-mat]].

[31] S. S. Gubser and I. Mitra, "Double-trace operators and one-loop vacuum energy in AdS/CFT", Phys. Rev. D 67, 064018 (2003) arXiv:hep-th/0210093]; S. S. Gubser and I. R. Klebanov, "A universal result on central charges in the presence of double-trace deformations", Nucl. Phys. B 656, 23 (2003) arXiv:hep-th/0212138; T. Hartman and L. Rastelli, "Doubletrace deformations, mixed boundary conditions and functional determinants in AdS/CFT", JHEP 0801, 019 (2008) arXiv:hep-th/0602106.

[32] Tian Zhang, unpublished notes. 
[33] S. Catterall, "Topological gravity on the lattice", arXiv:1003.5202 hep-lat]]; S. Catterall, "First results from simulations of supersymmetric lattices", JHEP 0901040 (2009), arXiv:0811.1203 hep-lat]]; S. Catterall, D. Ferrante, A. Nicholson, "de Sitter gravity from lattice gauge theory", arXiv:0912.5525[hep-lat]]; P. H. Damgaard, S. Matsuura, "Classification of Supersymmetric Lattice Gauge Theories by Orbifolding", JHEP 0707, 051 (2007), arXiv:0704.2696[hep-lat]].

[34] S. M. Carroll, "Spacetime and Geometry: An Introduction to General Relativity," Publisher: Benjamin Cummings (2003). 